OPEN ACCESS

Edited by:

Joaquin Bautista-Gallego,

Rovira i Virgili University, Spain

Reviewed by:

Raffaele Coppola,

University of Molise, Italy

Andre Gerald Buret,

University of Calgary, Canada

Geeta Shukla,

Panjab University, Chandigarh, India

${ }^{*}$ Correspondence:

Philippe Grellier

grellier@mnhn.fr

${ }^{\dagger}$ These authors have contributed equally to this work.

Specialty section:

This article was submitted to

Food Microbiology,

a section of the journal

Frontiers in Microbiology

Received: 18 July 2016

Accepted: 31 August 2016 Published: 27 September 2016

Citation:

Travers M-A, Sow C, Zirah S,

Deregnaucourt $C$, Chaouch $S$, Queiroz RML, Charneau S, Allain T,

Florent I and Grellier P (2016) Deconjugated Bile Salts Produced by Extracellular Bile-Salt Hydrolase-Like

Activities from the Probiotic Lactobacillus johnsonii La1 Inhibit Giardia duodenalis In vitro Growth

Front. Microbiol. 7:1453.

doi: 10.3389/fmicb.2016.01453

\section{Deconjugated Bile Salts Produced by Extracellular Bile-Salt Hydrolase-Like Activities from the Probiotic Lactobacillus johnsonii La1 Inhibit Giardia duodenalis In vitro Growth}

\author{
Marie-Agnès Travers ${ }^{1 \dagger}$, Cissé Sow ${ }^{2 \dagger}$, Séverine Zirah ${ }^{2}$, Christiane Deregnaucourt ${ }^{2}$, \\ Soraya Chaouch ${ }^{2}$, Rayner M. L. Queiroz ${ }^{3}$, Sébastien Charneau ${ }^{3}$, Thibault Allain ${ }^{2,4}$, \\ Isabelle Florent ${ }^{2}$ and Philippe Grellier ${ }^{2 *}$
}

'Laboratoire de Génétique et Pathologie des Mollusques Marins, Unité SG2M, IFREMER, La Tremblade, France, ${ }^{2}$ MCAM UMR 7245, Muséum National d'Histoire Naturelle, Centre National de la Recherche Scientifique, Sorbonne Universités, Paris, France, ${ }^{3}$ Department of Cell Biology, Institute of Biology, University of Brasilia, Brasilia, Brazil, ${ }^{4}$ UMR 1319, Commensal and

Probiotics-Host Interactions Laboratory, INRA, AgroParisTech, Jouy en Josas, France

Giardiasis, currently considered a neglected disease, is caused by the intestinal protozoan parasite Giardia duodenalis and is widely spread in human as well as domestic and wild animals. The lack of appropriate medications and the spread of resistant parasite strains urgently call for the development of novel therapeutic strategies. Host microbiota or certain probiotic strains have the capacity to provide some protection against giardiasis. By combining biological and biochemical approaches, we have been able to decipher a molecular mechanism used by the probiotic strain Lactobacillus johnsonii La1 to prevent Giardia growth in vitro. We provide evidence that the supernatant of this strain contains active principle(s) not directly toxic to Giardia but able to convert non-toxic components of bile into components highly toxic to Giardia. By using bile acid profiling, these components were identified as deconjugated bile-salts. A bacterial bile-salt-hydrolase of commercial origin was able to mimic the properties of the supernatant. Mass spectrometric analysis of the bacterial supernatant identified two of the three bile-salt-hydrolases encoded in the genome of this probiotic strain. These observations document a possible mechanism by which L. johnsonii La1, by secreting, or releasing BSH-like activity(ies) in the vicinity of replicating Giardia in an environment where bile is present and abundant, can fight this parasite. This discovery has both fundamental and applied outcomes to fight giardiasis, based on local delivery of deconjugated bile salts, enzyme deconjugation of bile components, or natural or recombinant probiotic strains that secrete or release such deconjugating activities in a compartment where both bile salts and Giardia are present.

Keywords: giardiasis, lactobacilli, probiotics, microbiota, bile salts, anti-giardial activity, choloylglycine bile acid hydrolase 


\section{INTRODUCTION}

Giardia duodenalis is a parasitic protozoa responsible for giardiasis, a disease characterized by acute or chronic intestinal malabsorption, diarrhea, weight loss, dehydration, and abdominal pain in humans and a variety of vertebrates. It is one of the most common intestinal parasites, with 200-300 million human cases estimated worldwide per year (Lane and Lloyd, 2002). Giardiasis has high veterinary impact and high impact on public health, and is responsible for human morbidity, especially causing nutritional deficiencies in children in developing countries (Lane and Lloyd, 2002; Ali and Hill, 2003; Veenemans et al., 2011). Developed countries are also concerned by giardiasis; outbreaks have been associated with drinking water contamination resulting from runoff of contaminated soils by rain falls, agricultural practices, and sewage treatment plant dysfunctions (Mons et al., 2009; Baldursson and Karanis, 2011).

Giardia duodenalis exists under two development forms, a resistant form called cyst, responsible for the transmission of the parasite between vertebrate hosts, and an active form called trophozoite, that replicates within intestinal tracts of hosts. Cysts enter vertebrate hosts via several routes such as food, water, or fomites contaminated by feces from infected hosts. Cysts remain infective for months in environmental waters and are infectious at low doses (10-100 cysts; Lane and Lloyd, 2002). Upon parasite excystation, newly formed trophozoites are released and colonize the surface of the intestinal barrier in the upper small intestine (duodenum). The duodenum constitutes a very specific microenvironment where chyme from the stomach, bile produced from the liver and stored in the gall bladder, and digestive enzymes from the pancreas pour in. After encystation in the lower part of the small intestine, parasites exit the host in the feces (Lane and Lloyd, 2002; Ali and Hill, 2003; Ankarklev et al., 2010).

The pathophysiology of giardiasis is multifactorial and comprises damages to the host's mucosal surface such as diffuse microvillous shortening and a reduction of villus-crypt ratios. An increase of epithelial permeability, which might lead to a bacterial translocation is also observed as well as an impairment of disacharridase activity of digestive enzymes (Cotton et al., 2011; Buret et al., 2015). These changes may be due as much to factors of the host as to those of the parasite. In recent studies, a post-infectious link has been established between Giardia infection and intestinal- and extra-intestinal disorders such as inflammatory bowel syndrome and chronic fatigue syndrome (Halliez and Buret, 2013; Ventura et al., 2004). The drug of choice for treating giardiasis remains metronidazole, a 5-nitroimidazole (Leitsch et al., 2011). However, drug side effects (vertigo, nausea, vomiting, anorexia, and dizziness; Gardner and Hill, 2001) and the occurrence of drug resistant strains (Upcroft and Upcroft, 2001; Lalle, 2010; Tian et al., 2010; Ansell et al., 2015) make research on alternative therapeutic strategies necessary.

Abbreviations: BSH, bile salt hydrolase; GC, glycocholate; GCDC, glycochenodeoxycholate; GDC, glycodeoxycholate; KM, TYI-S-33 Keiser' medium; KM-FCS, KM with fetal calf serum; MTYI, modified TYI-S-33; NEFA, non-esterified fatty acids; TC, taurocholate; TCDC, taurochenodeoxycholate; TDC, taurodeoxycholate.
In this context, it is now widely recognized that the intestinal microbiota plays a role in the protection of the host against the gut colonization by pathogens (Singer and Nash, 2000; Travers et al., 2011; Kamada et al., 2013; Bartelt and Sartor, 2015). Different mechanisms may be responsible for these protective effects such as competition for pathogen ecological niches, competition for nutritional substrates, production of antimicrobial compounds, and enhancement of the innate and adaptive host immune responses (Tancrède, 1992; Kamada et al., 2013). Works highlighted the importance of intestinal bacteria in G. duodenalis infection (Singer and Nash, 2000). Evidence suggests that the bacterium Enterococcus faecium contributes to the clearance of $G$. duodenalis by enhancing the host immune response (Benyacoub et al., 2005). Additionally, the probiotic bacterium Lactobacillus johnsonii La1 antagonizes G. duodenalis establishment in rodent models (Humen et al., 2005) and extracellular factors of L. johnsonii La1 arrest the in vitro growth of $G$. duodenalis in the G1 phase indicating that this bacterium may directly affect parasite replication (Pérez et al., 2001). A better knowledge of the molecular interactions between G. duodenalis and the duodenal microbiota could thus open new chemotherapeutic strategies based on the inhibition of specific parasite enzymes or processes. L. johnsonii Lal constitutes a model of choice to study the molecular crosstalks between $G$. duodenalis and probiotic bacteria such as lactobacilli, which are one of the most common bacteria in the duodenum. AntiGiardia activity was clearly demonstrated in vivo and in vitro (Pérez et al., 2001; Humen et al., 2005) and the genomes of L. johnsonii La1 and G. duodenalis have been sequenced (Pridmore et al., 2004; Morrison et al., 2007; Franzén et al., 2009).

In the present paper, we investigated the molecular mechanisms involved in the in vitro anti-Giardia activity of extracellular factors of the probiotic L. johnsonii La1. G. duodenalis can be cultured in vitro (Keister, 1983). Bile was historically added into Giardia culture medium to promote its in vitro growth, even if this gain in generation time could be reduced at high bile and bile salt concentrations (Farthing et al., 1983, 1985). Parasites can internalize bile lipids as metabolites or source of phospholipids for membrane biosynthesis (Farthing et al., 1985; Halliday et al., 1995; Das et al., 1997). Conjugated bile salts have also a role in promoting encystation (Gillin, 1987; Gillin et al., 1989). We found that the parasite growth inhibition by $L$. johnsonii Lal culture spent supernatant is mediated by secreted bacteria bile-salt hydrolase-like activity(ies) that generate(s) deconjugated bile salts from bile present in the culture medium. Unlike conjugated bile salts, the main components of bile, deconjugated bile salts were found to be toxic to Giardia.

\section{MATERIALS AND METHODS Products and Preparation of Stock Solutions}

Bovine bile (reference B3883, Sigma-Aldrich and reference 212820, Difco, BD Diagnostic Systems) was prepared at $100 \mathrm{mg} / \mathrm{ml}$ stock solution in distilled water, sterilized 
through $0.2 \mu \mathrm{m}$ filters and stored at $-20^{\circ} \mathrm{C}$. Pure bile salts: glycocholate (GC), taurocholate (TC), glycodeoxycholate (GDC), taurodeoxycholate (TDC), glycochenodeoxycholate (GCDC), and taurochenodeoxycholate (TCDC), bile salt mix or pure deconjugated bile salts: cholate (C), deoxycholate (DC), and chenodeoxycholate (CDC), and fusidic acid were from Sigma-Aldrich and dissolved in distilled water to $12 \mathrm{mg} / \mathrm{ml}$ stock solutions, filtered through $0.2 \mu \mathrm{m}$ pores and kept at $-20^{\circ} \mathrm{C}$. Choloylglycine Bile Acid Hydrolase or Bile Salt Hydrolase (BSH, EC 3.5.1.24) from Clostridium perfringens (reference C4018, Sigma-Aldrich) was prepared at $10 \mathrm{U} / \mathrm{ml}$ in distilled water and stored at $-20^{\circ} \mathrm{C}$. Fetal calf serum (FCS, reference A15-101, PAA Laboratories, GE Healthcare), and bovine calf serum (reference B9433, Sigma-Aldrich) were used. The NEFA-C kit used for quantitative determination of non-esterified fatty acids (NEFAs) was from Biolab, WAKO Diagnostics.

\section{Cell Culture of Giardia duodenalis}

Trophozoites of G. duodenalis strains WB (clone C6, ATCC 30957) and HP1 (Portland-1, gift of Jan Tackezy, Charles University of Prague, Czech Republic) from assemblage A were routinely maintained in axenic culture in TYI-S-33 Keister's medium (KM) as previously described (Keister, 1983). Parasites were grown in $15 \mathrm{ml}$ tubes filled with $12 \mathrm{ml}$ culture medium. They were regularly subcultured at a density of $5 \times 10^{4}$ cells per tube $(12 \mathrm{ml})$ from log phase parasites chilled on ice for $10 \mathrm{~min}$ and centrifuged at $700 \times g, 5 \mathrm{~min}$. For bioassays, parasites were maintained in culture in $\mathrm{KM}$ adjusted to $\mathrm{pH}$ 6.0, supplemented with $10 \%$ heat-inactivated FCS (Paget et al., 2004) and $0.6 \mathrm{~g} / \mathrm{L}$ bovine bile (Carnaby et al., 1994).

\section{Culture of Lactobacillus johnsonii La1 and Production of Bacterial Supernatant}

L. johnsonii La1 (CNRZ 1897, NCC533) was kindly provided by Pascal Quénée (INRA Jouy en Josas, Equipe Atalis, France) and was isolated from LC1 product in 1996 (Chambourcy, France). The presence of $L$. johnsonii Lal prophage, as previously described (Ventura et al., 2004; Denou et al., 2008), was checked by PCR using two primer pairs: $1 \mathrm{DF} / 1 \mathrm{~B} 2 \mathrm{R}$ resulting in a 1500 bp amplicon for L. johnsonii "La1-like" (with the prophage) and $1 \mathrm{EF} / 1 \mathrm{~B} 2 \mathrm{R}$ resulting in a $268 \mathrm{bp}$ amplicon as a positive control for L. johnsonii strains. PCR reactions were run in a final volume of $25 \mu \mathrm{l}$ containing $200 \mathrm{ng}$ of bacterial DNA extracted with a standard protocol (Zhong et al., 1998), $200 \mathrm{nM}$ of each primer (1DF: AGA CTT TTT GGC AGG CAA AGG, 1EF: ACA AAC CAC CAG TGC CTA AGG, 1B2R: GCT CTT CGA GAT CAC TGG GC), $200 \mathrm{nM}$ of dNTP, and $1 \mathrm{U}$ of Taq DNA polymerase (NEB M0273S). Reactions were initiated with an initial denaturation for $5 \mathrm{~min}$ at $95^{\circ} \mathrm{C}$ followed by 35 cycles at $95^{\circ} \mathrm{C}$ for $30 \mathrm{~s}, 50^{\circ} \mathrm{C}$ for $30 \mathrm{~s}$ and $72^{\circ} \mathrm{C}$ for $1 \mathrm{~min}$. PCR products were visualized on $0.8 \%$ agarose gel containing $0.5 \mathrm{ng} / \mu \mathrm{l}$ of BET and images were captured with a camera coupled with Biocapt software, Vilber Lourmat.

L. johnsonii La1 stock cultures were kept frozen in de Man Rogosa Sharpe (MRS) Broth (Sigma-Aldrich) media with $15 \%$ glycerol. Bacteria were subcultured in MRS or modified TYI-S-33 medium (MTYI) (Pérez et al., 2001) and incubated anaerobically for $12-18 \mathrm{~h}$ at $37^{\circ} \mathrm{C}$. Bacteria were subsequently grown in MTYI or KM supplemented with or without $10 \%$ heat-inactivated FCS for $12-16 \mathrm{~h}$ in the presence or the absence of $0.6 \mathrm{~g} / \mathrm{L}$ bovine bile. After centrifugation $(3000 \times$ $g, 10 \mathrm{~min}$ ) and $0.2 \mu \mathrm{m}$ filtration, the $\mathrm{pH}$ of the L. johnsonii La1 supernatant was adjusted to 6.0 with $5 \mathrm{~N} \mathrm{NaOH}$. For some experiments, $\mathrm{pH}$ was adjusted to $6.2,6.7,6.9$, or 7.2. Appropriated controls were prepared as follows: lactic acid produced during growth was quantified from aliquots of supernatants (Enzytec ${ }^{\mathrm{TM}}$ kit, R-Biopharm) and an equivalent amount of lactic acid was added to fresh medium before $\mathrm{pH}$ adjustment.

\section{In vitro G. duodenalis Growth Inhibition Assay}

One milliliter of trophozoite suspension $\left(1 \times 10^{5}\right.$ parasites $/ \mathrm{ml}$ in $\mathrm{KM}, \mathrm{pH}$ 6, supplemented with 10\% FCS without bovine bile) was mixed with either $500 \mu \mathrm{l}$ of $L$. johnsonii La1 supernatant or one, or two, units of commercial bile salt hydrolase $(\mathrm{BSH})$ from C. perfringens, chromatography purified fractions or appropriate control medium. These suspensions were further mixed with different concentrations of either bovine bile, mixed bile salts, conjugated, or deconjugated bile salts or appropriate control medium. Samples were then incubated for $24 \mathrm{~h}$ at $37^{\circ} \mathrm{C}$ and chilled on ice for $10 \mathrm{~min}$ to dislodge trophozoites from tube wall. Trophozoites were counted using a Malassez cell chamber and were considered alive when parasites showed the typical pear shape and signs of flagella mobility (Video S1). Morphologically altered parasites with non-mobile flagella were considered "dead" (Video S2). Estimates of parasite viability on these criteria correlate with parasite viability measured by propidium iodide staining and flow cytometry analysis (Barbosa et al., 2008; Figure S1). Multiplication factor [i.e., number of total trophozoites at the end of the experiment/number of trophozoites at time zero], survival rate [i.e., (number of living cells/total number of trophozoites $) \times 100]$, and inhibition percentage [i.e., (100-number of living cells in the presence of tested compounds/number of living cells in control) $\times 100]$ were calculated.

\section{Partial Purification of Active Fractions from L. johnsonii La1 Supernatants by Gel Filtration}

Supernatants from L. johnsonii Lal cultures in KM, adjusted to $\mathrm{pH}$ 6.0, were concentrated up to 30 -fold by ultrafiltration using the $10 \mathrm{kDa}$ Centriprep centrifugal filter unit (Millipore). After $0.2 \mu \mathrm{m}$ filtration, the concentrated supernatants were loaded onto a Sephacryl S300 column 16/100 (GE Healthcare), previously equilibrated with $20 \mathrm{mM}$ ammonium sulfate $(\mathrm{pH}$ 6.0 ), in a cold room and were eluted with the same buffer at a flow rate of $2.0 \mathrm{ml} / \mathrm{min}$. Effluent fractions of $12 \mathrm{ml}$ were collected, concentrated 4 -fold by ultrafiltration on a $10 \mathrm{kDa}$ Centriprep and tested for in vitro Giardia growth inhibition in the presence of bovine bile or bile salts. Fractions obtained by similar processing of elution buffer alone 
and control media containing lactic acid (see above) were used as controls. Column calibration was carried out with ribonuclease A $(13.7 \mathrm{kDa})$ and bovine serum albumin $(67$ $\mathrm{kDa})$.

\section{Characterization of the Inhibitory Molecule(s) in L. johnsonii La1 Supernatant}

The molecular size of inhibitory molecule(s) present in L. johnsonii Lal supernatant was assessed by ultrafiltration using 10,30 , and $50 \mathrm{kDa}$ Centriprep centrifugal filter units. Thermal stability was tested by heating the bacterial supernatant to $90^{\circ} \mathrm{C}$ for $10 \mathrm{~min}$. Preservation of L. johnsonii La1 supernatant activity upon dialysis was checked by dialyzing twice (at $4^{\circ} \mathrm{C}$ for 2 and $15 \mathrm{~h}$, respectively) the supernatant against 100 volumes of KM supplemented with 10\% FCS or against $\mathrm{GKN}$ solution $(\mathrm{NaCl}, 8 \mathrm{~g} / \mathrm{l} ; \mathrm{KCl}, 0.4 \mathrm{~g} / \mathrm{l} ;$ glucose, $2 \mathrm{~g} / \mathrm{l} ; \mathrm{NaH}_{2} \mathrm{PO}_{4}, \mathrm{H}_{2} \mathrm{O}, 0.69 \mathrm{~g} / \mathrm{l} ; \mathrm{Na}_{2} \mathrm{HPO}_{4}, 1.57 \mathrm{~g} / \mathrm{l} ; \mathrm{pH} 7.2-$ 7.4) (Pérez et al., 2001) using a dialysis membrane with a molecular weight cut-off of $3.5 \mathrm{kDa}$ (Spectrum Laboratories). The dialyzed supernatant was then filtrated through a $0.2 \mu \mathrm{m}$ membrane and kept frozen at $-80^{\circ} \mathrm{C}$ prior to G. duodenalis inhibition assays.

The biochemical nature of inhibitory molecule(s) was investigated by preincubating the 5-fold concentrated L. johnsonii La1 supernatant obtained by ultrafiltration $(>10$ $\mathrm{kDa}$ ) with different enzymes coupled to beads. Briefly, proteinase $\mathrm{K}$ (Invitrogen), pronase and catalase (Merck) were coupled to $\mathrm{CNBr}$-activated Sepharose ${ }^{\mathrm{TM}} 4 \mathrm{~B}$ beads (GE Healthcare) following manufacturer's instructions. Five milliliters of the 5-fold concentrated L. johnsonii La1 supernatant or the 5 -fold concentrated fresh control medium were incubated for $6 \mathrm{~h}$ at room temperature (RT) in the presence of $100 \mu \mathrm{l}$ of packed enzyme-coupled beads. Beads were removed by centrifugation $(4000 \times g, 5 \mathrm{~min})$ before parasite growth inhibition assays.

\section{Measurement of Free Fatty Acids}

To assess the presence of free fatty acids in the complex medium inducing G. duodenalis growth inhibition, FCS, bile, and L. johnsonii Lal supernatant were analyzed for non-esterified fatty acid (NEFA) content either alone or in combination in KM. Respective concentrations of the components in samples were as follows: FCS $10 \%(\mathrm{v} / \mathrm{v})$, bile $0.6 \mathrm{~g} / \mathrm{L}$ and $L$. johnsonii La1 supernatant $33.3 \%(\mathrm{v} / \mathrm{v})$ final concentration in $\mathrm{KM}, \mathrm{pH}$ 6. Samples $(0.5 \mathrm{ml})$ were kept on ice before being incubated for $24 \mathrm{~h}$ at $37^{\circ} \mathrm{C}$ in the presence of $4.8 \times 10^{4}$ trophozoites or without parasites. At the end of the incubation period, tubes were chilled on ice, centrifuged at $700 \times g, 10 \mathrm{~min}$ at RT. The supernatants were then taken and frozen at $-80^{\circ} \mathrm{C}$ before NEFA measurement. Numbers of living and "dead" trophozoites (as defined above) were determined using a Malassez cell chamber. NEFAs were quantified using the spectrometric NEFA-C kit method, based on Duncombe (1964) and Itaya and Ui (1965) studies, following manufacturer's instructions. Oleic acid was used as a standard and NEFAs were expressed as oleic acid equivalents (Eq).

\section{Bile Salt Hydrolase (BSH) Activity Assays of Chromatography Fractions}

After gel filtration, the eluted fractions were concentrated 10-fold by dialysis against $20 \mathrm{mM}$ ammonium acetate buffer containing $2 \mathrm{M}$ sucrose, $\mathrm{pH} 6.0$, using a $3.5 \mathrm{kDa}$ molecular weight cut-off membrane (Spectrum Laboratories). Bile salt GDC was used for enzymatic assays. BSH activity was monitored by measuring glycine liberation from conjugated bile salts following the protocol described by Grill et al. (2000). Briefly $100 \mu l$ of effluent fractions or 1 unit of BSH or elution buffer were mixed with $100 \mu \mathrm{l}$ of $2.4 \mathrm{~g} / \mathrm{l}$ of GDC and incubated for $24 \mathrm{~h}$ at $37^{\circ} \mathrm{C}$. Controls were performed in the absence of the bile salt. The enzymatic reaction was stopped by addition of an equal volume of $15 \%$ TCA. Precipitated proteins were pelleted by centrifugation at $20,000 \times g$ for $15 \mathrm{~min}$ and the supernatant was carefully removed. To $80 \mu \mathrm{l}$ of supernatant, $680 \mu \mathrm{l}$ of $0.3 \mathrm{M}$ borate buffer, $1 \%$ SDS, $\mathrm{pH} 9.5$ and $80 \mu \mathrm{l}$ of $0.3 \%$ picrylsulfonic acid solution (SigmaAldrich) were added. The mixture was incubated for $30 \mathrm{~min}$ in the dark and $800 \mu \mathrm{l}$ of $1 \mathrm{mM} \mathrm{HCl}$ was added to stop the reaction. Glycine concentration was measured at $416 \mathrm{~nm}$ using an Uvikon spectrophotometer 930 (Kontron Instruments). A standard curve was established using free glycine (Sigma-Aldrich).

\section{LC/ESI-MS Analysis of Modifications of Bile Components by L. johnsonii La1 Supernatants}

L. johnsonii La1 supernatant prepared in $\mathrm{KM}, \mathrm{pH}$ 6.0, supplemented with $10 \%$ FCS and $1 \mathrm{~g} / \mathrm{L}$ bovine bile was incubated overnight at $37^{\circ} \mathrm{C}$. Two different bile batches and two different L. johnsonii La1 supernatant preparations were tested. Controls constituted of $\mathrm{KM}$ alone and heat-treated L. johnsonii La1 supernatant $\left(90^{\circ} \mathrm{C}, 10 \mathrm{~min}\right)$. The sample preparation and LCMS protocol were adapted from the conditions reported in the literature on the LC-MS analysis of bile (Mitamura et al., 2011) and bile acids (Perwaiz et al., 2001; Griffiths and Sjövall, 2010). After incubation, the samples were diluted 4 -fold in Milli-Q water and subjected to solid-phase extraction using Oasis ${ }^{\circledR}$ HLB cartridges (30 mg solid phase). After conditioning with $3 \mathrm{ml}$ methanol and $3 \mathrm{ml}$ Milli-Q water, the cartridges were loaded with $1 \mathrm{ml}$ of the 4 -fold diluted samples, washed with $2 \mathrm{ml}$ Milli-Q water and eluted with $2 \mathrm{ml}$ methanol. The eluted fractions were vacuum dried and resuspended in $500 \mu \mathrm{l}$ Milli-Q water/acetonitrile 90:10 (v/v). $5 \mu \mathrm{l}$ of each resuspended sample was analyzed by liquid chromatography coupled electrospray ionization-mass spectrometry (LC/ESI-MS) on an Ultimate U3000 chromatographic system (Thermo Scientific) connected to a Q-STAR Pulsar Qq-TOF mass spectrometer equipped with an ionspray source (Sciex). The LC separation was achieved by an Interchrom Strategy C18-2 micro column $(5 \mu \mathrm{m}, 150 \times$ $1 \mathrm{~mm}, 100 \AA$, Interchim). The elution gradient was $10 \%$ mobile phase B (acetonitrile) to $70 \%$ B against mobile phase A ( $5 \mathrm{mM}$ ammonium formate/formic acid, $\mathrm{pH}$ 6) over $45 \mathrm{~min}$, at a flow rate of $40 \mu \mathrm{l} / \mathrm{min}$. The MS data were acquired in negative mode in the range $m / z$ 250-1200. Each LC/ESI-MS experiment was conducted twice. Data-dependent LC/ESI-MS/MS experiments were also conducted on each sample, alternating 1-s full-scan 
MS followed by 2-s product ion collision induced dissociation of the major ions detected at the first step, using a $-50 \mathrm{~V}$ collision voltage.

The raw LC/ESI-MS data were converted into Network Commun Data Form (NetCDF) using the translation tool provided by Sciex and processed with the XCMS package (Smith et al., 2006), a software implemented in the freely available $\mathrm{R}$ environment (www.r-project.org). This analysis allows automatic retention time alignment, matched filtration, peak detection, and peak matching.

\section{Proteomic Analysis of $L$. johnsonii La1 Supernatant}

Sodium lauroyl sarcosinate (NLS, 0.1\%, Sigma-Aldrich) was added to L. johnsonii Lal supernatant. After mixing, trichloroacetic acid (TCA) was added to a final $7.5 \%$ concentration and the solution was precipitated on ice overnight. Mixed protein-detergent precipitate was collected by centrifugation $\left(10,000 \times g, 10 \mathrm{~min}, 4^{\circ} \mathrm{C}\right)$. The supernatant was carefully removed and the pellet was washed twice with $2 \mathrm{ml}$ of precooled tetrahydrofuran (Sigma-Aldrich). The pellet was then resuspended in $8 \mathrm{M}$ urea in $20 \mathrm{mM}$ triethylammonium bicarbonate and incubated for $1 \mathrm{~h}$ at $20^{\circ} \mathrm{C}$ with $20 \mathrm{mM}$ DTT, followed by incubation with $50 \mathrm{mM}$ iodacetamide for $1 \mathrm{~h}$ at $20^{\circ} \mathrm{C}$ in the dark. The sample was incubated with $0.05 \mathrm{U}$ of endoproteinase Lys-C (Wako Pure Chemical Industries) for $18 \mathrm{~h}$ at RT. Then, the samples were diluted with $20 \mathrm{mM}$ triethylammonium bicarbonate to a final concentration of $1 \mathrm{M}$ urea and trypsin (Promega) digestion was performed with $2 \mu \mathrm{g}$ of enzyme for $4 \mathrm{~h}$ at $20^{\circ} \mathrm{C}$ and terminated with a final concentration of $0.5 \%$ trifluoroacetic acid. The sample was passed sequentially through two home-made Poros Oligo-R3 (PerSeptive Biosystems) microcolumns packed $(\sim 1 \mathrm{~cm})$ on $\mathrm{p} 200$ tips over 3MM C18 material plug. Loaded resin was washed with $100 \mu \mathrm{l} 0.1 \%$ trifluoroacetic and peptides were eluted with $100 \mu \mathrm{l} 70 \%$ acetonitrile/ $0.1 \%$ trifluoroacetic, then $20 \mu \mathrm{l} 100 \%$ acetonitrile. The desalted sample was dried down, resuspended in $50 \%$ acetonitrile, dried, and stored at $-80^{\circ} \mathrm{C}$. Part of the sample (10\%) was collected for amino acid analysis using a Biochrom 30 amino acid analyzer.

Samples ( $3 \mu \mathrm{g}$ per run) were analyzed by an EASY-nano LC system (Proxeon Biosystems) coupled online to an LTQOrbitrap Velos mass spectrometer (Thermo Scientific). Peptides were loaded onto a $18 \mathrm{~cm}$ fused silica emitter $(75 \mu \mathrm{m}$ inner diameter) packed in-house with reverse phase capillary column ReproSil-Pur C18-AQ $3 \mu \mathrm{m}$ resin (Dr. Maisch GmbH) and eluted using a gradient from $100 \%$ phase $\mathrm{A}(0.1 \%$ formic acid $)$ to $35 \%$ phase B (0.1 formic acid, 95\% acetonitrile) for $180 \mathrm{~min}$, 35 to $100 \%$ phase B for $5 \mathrm{~min}$ and $100 \%$ phase B for $8 \mathrm{~min}$ (a total of $23 \mathrm{~min}$ at $250 \mathrm{nl} / \mathrm{min}$ ). After each run, the column was washed with $90 \%$ phase B and re-equilibrated with phase A. Mass spectra were acquired in positive mode applying data-dependent automatic survey MS scan and tandem mass spectra (MS/MS) acquisition. Each MS scan in the orbitrap (mass range of $\mathrm{m} / \mathrm{z}$ of 400-1800 and resolution 100,000) was followed by MS/MS of the 15 most intense ions in the LTQ. Fragmentation in the LTQ was performed by collision-induced dissociation and selected sequenced ions were dynamically excluded for $25 \mathrm{~s}$.

Raw data were viewed in Xcalibur v.2.1 (Thermo Scientific) and data processing was performed using Proteome Discoverer v.1.3 (Thermo Scientific). The generated raw files were submitted to searching using Proteome Discoverer with an in house Mascot v.2.3 algorithm against a database of proteins predicted from the L. johnsonii isolates NCC533 and FI9785 and the L. johnsonii prophage Lj965 (http://www.ncbi.nlm.nih.gov/bioproject). Contaminant proteins (human keratins, BSA and porcine trypsin) were also added to the database and all contaminant proteins identified were manually removed from the result lists. The searches were performed with the following parameters: ms accuracy $10 \mathrm{ppm}$, MS/MS accuracy 0.6 Da, trypsin digestion with one missed cleavage allowed, fixed carbamidomethyl modification of cysteine and variable modification of oxidized methionine and N-terminal protein acetylation. The number of proteins, protein groups, and number of peptides were filtered for False Discovery Rates (FDR) less than 1\%. Only peptides with rank 1 and minimal of 2 peptides per protein were accepted for identification using Proteome Discoverer (Charneau et al., 2007; Queiroz et al., 2013).

\section{Statistical Analyses}

Significant differences between treatments were tested using the "Kruskal-Wallis" test in http://marne.u707.jussieu.fr/biostatgv/. Results were considered significant at $P<0.05$.

\section{RESULTS}

\section{The Anti-Giardia Growth Effect, Mediated by $L$. johnsonii La1 Supernatant, is Dependent upon Bovine Bile and More Specifically upon Bile Salts}

Pérez et al. (2001) showed that the inhibitory effect of L. johnsonii Lal supernatant upon in vitro $G$. duodenalis growth was $\mathrm{pH}$ dependent with a more pronounced inhibitory effect at $\mathrm{pH}$ 6 than $\mathrm{pH}$ 7. Change of culture medium $\mathrm{pH}$ had a slight effect on parasite proliferation and adhesion properties. We thus set up our culture assays at $\mathrm{pH}$ 6. FCS was used instead of adult bovine serum for better reproducibility of the assay. In our experimental conditions, parasite morphology, motility, and viability measured by propidium iodide staining were unaffected (Video S1, Figure S1).

Most media previously described to support G. duodenalis growth in vitro commonly contains bile as a supply for parasite cholesterol and fatty acid requirements (Farthing et al., 1985; Gillin et al., 1986; Halliday et al., 1995). In our hands, G. duodenalis trophozoites growth was observed in the absence of bovine bile. Bile, at the recommended concentration $(0.75 \mathrm{~g} / \mathrm{L})$ (Pérez et al., 2001), appeared to reduce trophozoite proliferation when added to KM-FCS (Figure 1A). We next confirmed the in vitro inhibitory effect of L. johnsonii La1 supernatant upon G. duodenalis growth. Interestingly, the inhibitory effect was only observed in the presence of bovine bile. No inhibitory effect was observed in the absence of bovine bile, even after $24 \mathrm{~h}$ of culture 

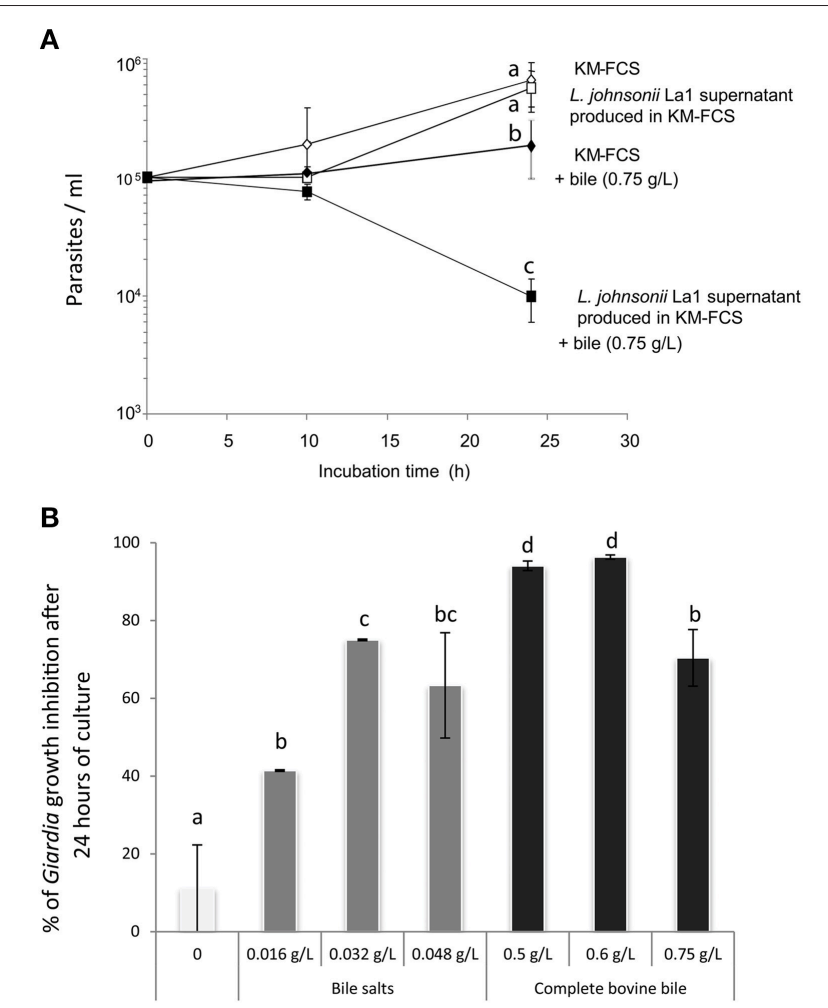

FIGURE 1 | (A) The inhibitory effect of $L$. johnsonii La1 supernatant on G. duodenalis growth is observed after $24 \mathrm{~h}$ in the presence of bovine bile. $\mathrm{G}$. duodenalis trophozoites were grown in KM-FCS with bovine bile $(0.75 \mathrm{~g} / \mathrm{L}$, final concentration) in the presence $(\boldsymbol{\square})$ or in the absence $(\boldsymbol{})$ of bacterial supernatant, or without bovine bile in the presence $(\square)$ or in the absence $(\diamond)$ of bacterial supernatant. The parasite concentration was estimated by counting live cells with a Malassez cell chamber. Values are the mean \pm SD of two independent experiments performed in triplicate. Letters indicate significant differences between treatments (Kruskall-Wallis, $p<0.05$ ). (B) G. duodenalis growth inhibition by $L$. johnsonii La1 supernatant depends on the presence of bile, more specifically of bile salts. G. duodenalis trophozoites in KM-FCS were incubated for $24 \mathrm{~h}$ with $L$. johnsonii La1 supernatant and various concentrations of mixed bile salts $(0.016,0.032,0.048 \mathrm{~g} / \mathrm{L}$, final concentration) or complete bovine bile $(0,0.5,0.6,0.75 \mathrm{~g} / \mathrm{L}$, final concentration). Growth inhibition values (\%) were normalized according to controls in lactic acid-acidified KM-FCS supplemented with similar concentrations of bovine bile or mixed bile salts. Values are the mean \pm SD of three independent experiments. Letters indicate significant differences between treatments (Kruskall-Wallis, $p<0.05$ ).

(Figure 1A). After $10 \mathrm{~h}$ in the presence of $0.75 \mathrm{~g} / \mathrm{L}$ of bovine bile, G. duodenalis trophozoite survival was slightly impacted by L. johnsonii Lal supernatant, but survival was largely affected after $24 \mathrm{~h}$ of contact $\left(9.10^{4}\right.$ and $1.10^{4}$ trophozoite/ml, respectively, Figure 1A, Video S2, Figure S1). Thus, a $24 \mathrm{~h}$ incubation timeperiod was retained for all subsequent inhibitory growth assays.

Maximal inhibitory effects of L. johnsonii Lal supernatant on parasite growth were observed either in the presence of $0.6 \mathrm{~g} / \mathrm{L}$ of complete bovine bile or in the presence of $0.032 \mathrm{~g} / \mathrm{L}$ of bile salt mix ( 96.3 and $75 \%$ of inhibition, respectively, by comparison with control media containing the same amount of lactic acid, bile or bile salt mix, Figure 1B, $p<0.05$ ). Similar inhibitory effects were observed using two isolates of G. duodenalis (WB and
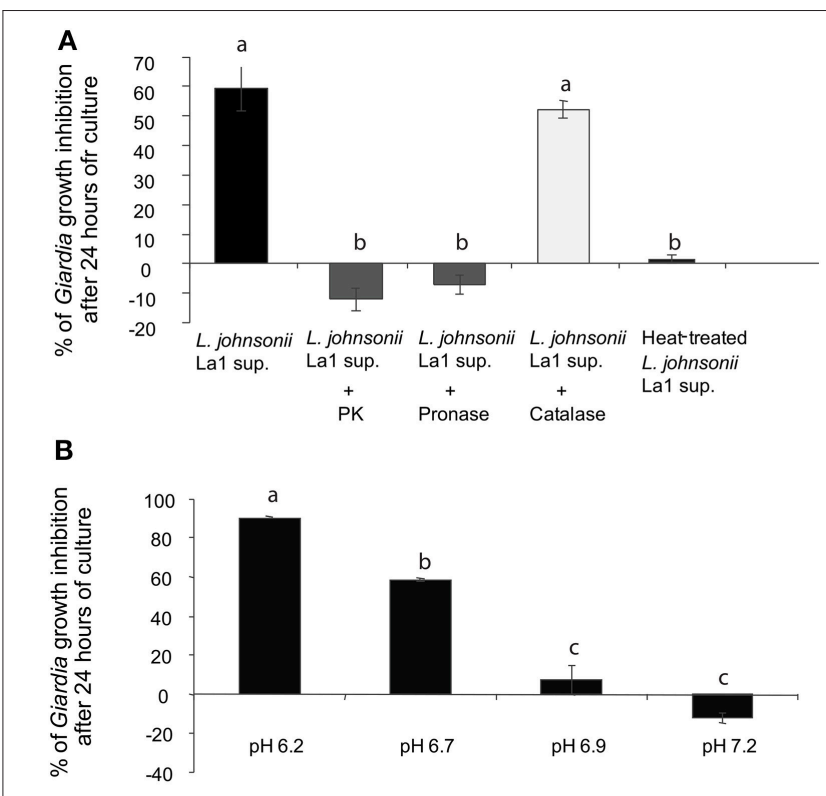

C

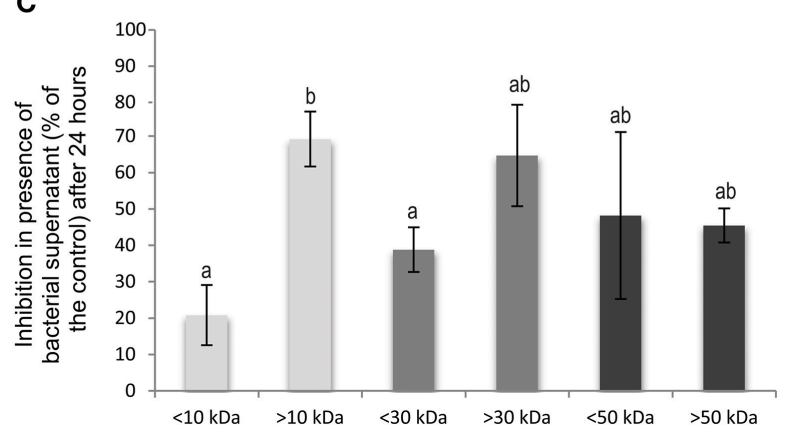

FIGURE 2 | G. duodenalis growth inhibition by L. johnsonii La1 supernatant is affected by supernatant incubation with proteases, heat-treatment and pH. (A) Supernatant of $L$. johnsonii La1 was incubated for $6 \mathrm{~h}$ with $1 \mathrm{mg}$ of immobilized proteases (L. johnsonii La1 supernatant: no protease treatment; L. johnsonii La1 supernatant + PK: treatment with proteinase K; L. johnsonii La1 supernatant + Pronase: treatment with pronase; $L$. johnsonii La1 supernatant + Catalase: treatment with catalase) or heated at $90^{\circ} \mathrm{C}$ for $10 \mathrm{~min}$ before $\mathrm{G}$. duodenalis growth inhibition assay. Growth inhibition (\%) was normalized according to matched control: lactic acid-adjusted MTYI medium incubated with protease-coupled beads or treated for $10 \mathrm{~min}$ at $90^{\circ} \mathrm{C}$. (B) Supernatant from L. johnsonii La1 in MTYI medium was adjusted to pH 6.2, 6.7, 6.9, or 7.2 before Giardia growth inhibition assays. Growth inhibition (\%) was normalized according to control, i.e., lactic acid-adjusted MTYI subsequently raised to $\mathrm{pH} 6.2,6.7,6.9$, or 7.2. Values are the mean \pm $\mathrm{SD}$ of three independent experiments. (C) Molecular weight determination of inhibitory compounds from $L$. johnsonii La1 supernatant. Supernatant from a culture of L. johnsonii La1 in KM-FCS was filtrated through 10, 30, or $50 \mathrm{kDa}$ MW cut-off membranes. Acidified KM-FCS alone was processed similarly. Fractions above and under respective thresholds were assayed for Giardia growth inhibition in the presence of bile $(0.5 \mathrm{~g} / \mathrm{L})$. Inhibition values (\%) were normalized according to KM-FCS controls. Data are the mean \pm SD of three independent experiments performed in triplicate. Letters indicate significant differences between treatments (Kruskal-Wallis, $p<0.05$ ).

HP1), two different commercial origins of complete bovine bile (Figure S2) and two different media compatible with bacteria and parasite growth: MTYI (Pérez et al., 2001) and KM-FCS (Paget 
et al., 2004). Unless otherwise indicated, subsequent in vitro $G$. duodenalis inhibition growth assays were performed using KM as medium at $\mathrm{pH} 6$ and $0.6 \mathrm{~g} / \mathrm{L}$ of complete bovine bile.

\section{Biochemical Characterization of Inhibitory Activity}

\section{L. johnsonii Extracellular Products Inhibiting Giardia Growth are of Peptidic Nature}

To biochemically characterize the inhibitory activity present in L. johnsonii Lal supernatant, the supernatant was treated with immobilized enzymes prior to contact with parasites. Trophozoite growth inhibition was totally abolished by proteinase $\mathrm{K}$ and pronase treatments, suggesting involvement of inhibitory factor(s) of peptidic nature (Figure 2A). Heattreatment also led to inactivation of $L$. johnsonii Lal supernatant inhibitory properties (Figure 2A). Additionally, in a $\mathrm{pH}$ range similar to the ones experienced by G. duodenalis in vivo (Biagini et al., 2001), a strong $\mathrm{pH}$ influence on the inhibitory activity was noticed, with the highest inhibition occurring at $\mathrm{pH} 6.2$ (Figure 2B).

Moreover, Pridmore et al. (2008) demonstrated L. johnsonii La1 supernatant anti-Salmonella activity was mediated by the toxic effects of hydrogen peroxide $\left(\mathrm{H}_{2} \mathrm{O}_{2}\right)$ and can be abolished by pretreatment with catalase. Therefore, we checked whether treatment of L. johnsonii Lal supernatant with catalase might prevent its anti-Giardia activity. Catalase pretreatment only slightly affected the inhibitory activity of L. johnsonii Lal supernatant on G. duodenalis (Figure 2A), invalidating the role of $\mathrm{H}_{2} \mathrm{O}_{2}$ in Giardia growth inhibition.

To assess whether $G$. duodenalis growth inhibition by L. johnsonii La1 supernatant might be due to toxic free fatty acids as demonstrated for G. duodenalis killing by human milk (Rohrer et al., 1986), FCS, bile, and L. johnsonii La1 supernatant were analyzed for NEFA content either alone or in combination, using the NEFA-C methodology (WAKO diagnostics). Then different samples were incubated for $24 \mathrm{~h}$ at $37^{\circ} \mathrm{C}$ with or without $\mathrm{G}$. duodenalis trophozoites and cell supernatants were analyzed for NEFAs. Survival and growth of the parasites in those different conditions were determined.

KM medium with FCS or L. johnsonii Lal supernatant, displayed NEFA concentrations of 37.7 and $39.5 \mu \mathrm{M}$ (oleic acid equivalents), respectively (Table 1). Co-incubation of bile with serum led to increased NEFA concentration $(101.2 \mu \mathrm{M})$ suggesting that the increase might result from improved exposure of protein-bound fatty acids from serum by the action of bile acids, thus facilitating substrate (fatty acid) recognition by enzymes from the NEFA kit. The highest concentration of NEFAs $(134.6 \mu \mathrm{M})$ was measured upon co-incubation of FCS with bile and L. johnsonii LA1 supernatant, as expected from summing their respective NEFA contents. It was verified that the presence of G. duodenalis had no noticeable effect on the NEFA content of the various samples (Table 1).

From the results in Table 1, it can be noted that normal parasite growth and survival (97.1\%) observed in KM-FCS-bile was totally inhibited by addition of $L$. johnsonii La1 supernatant which induced $100 \%$ of parasite death, although the NEFA concentration was increased by only 1.33 X (i.e., from 101.2 to $134.6 \mu \mathrm{M})$. This strongly suggested that NEFAs do not play a prominent role in Giardia inhibition by $L$. johnsonii Lal supernatant.

\section{L. johnsonii Extracellular Products Inhibiting Giardia Growth are $>10$ kDa Factor(s)}

Fractionation experiments of $L$. johnsonii Lal supernatant by ultrafiltration using different molecular weight cut-offs indicated that the L. johnsonii Lal supernatant inhibitory activity was due to molecule(s) bigger than $10 \mathrm{kDa}$, since the fraction $>10 \mathrm{kDa}$ showed a much higher $G$. duodenalis inhibition compared to the fraction $<10 \mathrm{kDa}$ (around 70 vs. $20 \%$ inhibition, respectively, $p<0.05$, Figure 2C). By performing a $30 \mathrm{kDa}$ threshold fractionation, the inhibitory activity concentrated mostly in the $>30 \mathrm{kDa}$ fraction (66\% of Giardia growth inhibition, even if not significantly different from the control, $p>0.05$, Figure 2C), however an inhibitory effect $(\sim 40 \%)$ was also observed with the $<30 \mathrm{kDa}$ fraction. A $50 \mathrm{kDa}$ threshold fractionation of $L$. johnsonii La1 supernatant was unable to segregate the inhibitory activity, i.e., 50 and $48 \%$ of Giardia growth inhibition were induced by $<50$ and $>50 \mathrm{kDa}$ fractions, respectively (Figure 2C).

Fractionation of $L$. johnsonii La1 supernatant was also realized by dialysis using a $3.5-\mathrm{kDa}$ cut-off membrane against KM-FCS or GKN buffer (Pérez et al., 2001). Parasite killing activity of L. johnsonii La1 supernatant in the presence of bile was fully recovered after dialysis, whatever the dialysis solution, indicating that no element crucial to the inhibitory activity was lost upon dialysis (Table 2). All together, these data indicated that $L$. johnsonii Lal supernatant factor(s) involved in the inhibitory activity display molecular weight $>$ to $10 \mathrm{kDa}$.

\section{L. johnsonii La1 Supernatant Modifies Bile Composition by Increasing Deconjugated Bile Salts that are Toxic to Giardia}

Since concomitant addition of bovine bile and L. johnsonii La1 supernatant to the culture medium leads to inhibition of $G$. duodenalis growth, we assessed whether bile composition might be modified by $L$. johnsonii Lal supernatant. Bile composition was investigated by LC/ESI-MS after $24 \mathrm{~h}$ of incubation with $L$. johnsonii La1 supernatant (Figures 3A,B). Impacted molecules were identified by their $\mathrm{m} / z \mathrm{MS} / \mathrm{MS}$ fragmentation pattern and comparison with standards. Comparison of bile salt profiles showed a decrease of conjugated salts (GC, TC, GDC, TDC, GCDC, TCDC) in favor of non-conjugated salts. $C$ and DC were the main statistically enhanced non-conjugated salts (Figure 4) and in a minor proportion, CDC. These modifications were not observed in presence of heat-treated $L$. johnsonii La1 supernatant (Figure 3C).

The inhibition of $G$. duodenalis growth by pure bile salts $(\mathrm{C}, \mathrm{DC}$, and $\mathrm{CDC})$ conjugated to glycine or taurine, or their deconjugated counterparts were investigated in the presence or the absence of L. johnsonii Lal supernatant (Table 3). In the absence of L. johnsonii Lal supernatant, glycyl-, or taurylconjugated salts and deconjugated $C$ showed no apparent toxicity at the concentrations tested $\left(\mathrm{IC}_{50}\right.$ values $\left.>400 \mu \mathrm{M}\right)$. In contrast, 
TABLE 1 | Involvement of non-esterified fatty acids in the G. duodenalis inhibition by L. johnsonii La1 supernatant.

\begin{tabular}{|c|c|c|c|}
\hline Incubation medium & $\begin{array}{c}\text { NEFAs ( } \mu \text { M Eq) in } \\
\text { presence of Giardia }\end{array}$ & $\begin{array}{c}\text { NEFAs ( } \mu \mathrm{M} \mathrm{Eq)} \mathrm{in} \mathrm{the} \\
\text { absence of Giardia }\end{array}$ & $\begin{array}{c}\text { Survival rate (\%) in the } \\
\text { presence of Giardia }\end{array}$ \\
\hline $\mathrm{KM}$ & 0 & $1.5 \pm 1.4$ & $21.4 \pm 15.7$ \\
\hline $\mathrm{KM}+\mathrm{FCS}$ & $37.7 \pm 4.1$ & $25.4 \pm 8.3$ & $94.7 \pm 4.3$ \\
\hline $\mathrm{KM}+$ L. johnsonii La1 supernatant & $39.5 \pm 6.8$ & $37.4 \pm 17.3$ & $50.0 \pm 20.7$ \\
\hline $\mathrm{KM}+\mathrm{FCS}+$ bile & $101.2 \pm 6.8$ & $87.7 \pm 5.1$ & $97.1 \pm 2.7$ \\
\hline KM + FCS + L. johnsonii La1 supernatant & $66.4 \pm 4.2$ & $60.1 \pm 15.7$ & $91.7 \pm 3.7$ \\
\hline KM + FCS + bile + L. johnsonii La1 supernatant & $134.6 \pm 5.5$ & $149.5 \pm 16.8$ & 0 \\
\hline
\end{tabular}

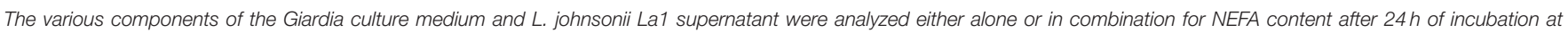

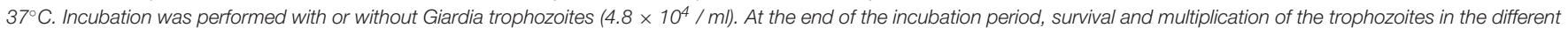

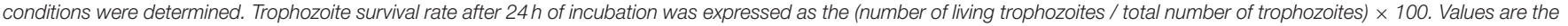
mean $\pm S D$ from three independent experiments. It was verified that NEFA contents after $24 \mathrm{~h}$ of incubation at $37^{\circ} \mathrm{C}$ were similarly independent of the presence of Giardia.

TABLE 2 | Dialysis through 3.5 kDa cut-off membrane does not inactivate the inhibitory activity of $L$. johnsonii La1 supernatant.

\begin{tabular}{|c|c|c|c|c|}
\hline & KM-FCS & $\begin{array}{l}\text { L. johnsonii } \\
\text { La1 supernatant }\end{array}$ & $\begin{array}{l}\text { L. johnsonii La1 supernatant } \\
\text { dialyzed against KM-FCS }\end{array}$ & $\begin{array}{l}\text { L. johnsonii La1 supernatant } \\
\text { dialyse against GKN }\end{array}$ \\
\hline \multicolumn{5}{|c|}{ GROWTH INHIBITION ASSAY IN THE ABSENCE OF BOVINE BILE } \\
\hline Multiplication factor & 3.8 & 3.2 & 3.7 & 2.4 \\
\hline Survival rate (\%) & 93.7 & 88.3 & 90.2 & 89.3 \\
\hline \multicolumn{5}{|c|}{ GROWTH INHIBITION ASSAY IN THE PRESENCE OF BOVINE BILE } \\
\hline Multiplication factor & 2.3 & 1.2 & 1.5 & 0.8 \\
\hline Survival rate (\%) & 92.9 & 0 & 0 & 0 \\
\hline
\end{tabular}

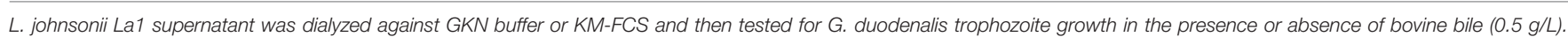
Non-dialyzed KM-FCS and L. johnsonii La1 supernatant were used as controls. Parasite multiplication factor was expressed as the number of total trophozoites / number of trophozoites

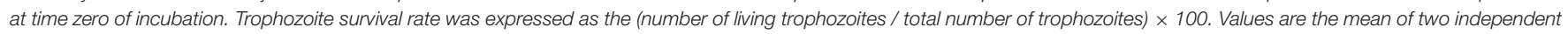
experiments performed in duplicate.

deconjugated DC, and CDC exhibited inhibitory effects on trophozoite growth with $\mathrm{IC}_{50}$ values of $132 \mu \mathrm{M}$ (DC) and $147 \mu \mathrm{M}$ (CDC). Interestingly, in the presence of $L$. johnsonii La1 supernatant, the conjugated bile salts $\mathrm{IC}_{50}$ values fell to a range of values similar to those measured for their pure deconjugated counterparts, i.e., 104 (GDC), 79 (TDC), 110, (GCDC), and $115 \mu \mathrm{M}$ (TCDC) (Table 3).

These results suggested that a deconjugating process mediated by L. johnsonii Lal supernatant component(s) and production of deconjugated bile salts might be responsible for the inhibitory effect of the association of bile with L. johnsonii La1 supernatant. Such a hypothesis is in line with the previous observation that fusidic acid, an antibiotic with a bile salt-like chemical structure, is toxic to Giardia (see Table 3, $\mathrm{IC}_{50}$ value $=26 \mu \mathrm{M}$ ) unless conjugated to taurine or glycine (Farthing and Inge, 1986).

\section{Potential Involvement of $L$. johnsonii La1 Bile-Salt Hydrolase(s) Activity in Bile-Mediated Giardia Inhibition Correlation between Bile Salt Hydrolase Activity and Anti-Giardial Properties after Fractionation of Extracellular Products}

It is known that the bile salt deconjugating process is mediated by 3-alpha, 7-alpha, 12-alpha-trihydroxy-5-beta-cholan24-oylglycine / taurine amidohydrolases (EC 3.5.1.24), also named choloylglycine / taurine hydrolases, conjugated bile acid hydrolases (CBAH), or BSH (Nair et al., 1967). These enzymes act on non-peptide carbon-nitrogen bonds, specifically in linear amides, releasing glycine and taurine from conjugated bile salts. Three genes (LJ0056, LJ1147, and LJ1412) encoding BSH-like enzymes have been predicted in the genome of L. johnsonii (Morrison et al., 2007). These enzymes are also predicted to be secreted, as indicated using SecretomeP (predictions of a non-classical i.e., not signal peptide triggered protein secretion), with theoretical full sizes of $34.9,36.3$, and $36.6 \mathrm{kDa}$ and secreted sizes of $29.8,31.0$, and $31.3 \mathrm{kDa}$, respectively.

To test the hypothesis of bacterial $\mathrm{BSH}$ involvement in the inhibitory activity of L. johnsonii Lal supernatant, the bacterial supernatant was fractionated by gel filtration chromatography on a Sephacryl S300 column, and eluted fractions were assayed for both parasite inhibition and bile salt deconjugating activity. A typical elution profile is shown in Figure 5A. Fractions were collected, tested for G. duodenalis growth inhibition and for BSHlike activity by measuring release of glycine from GDC. In our experimental conditions, 1 unit of the commercial C. perfringens $\mathrm{BSH}$ induced the release of $0.76 \mathrm{mM}$ glycine from $2.5 \mathrm{mM}$ GDC within $24 \mathrm{~h}$. Parasite growth inhibitory activity was reproducibly recovered from the first eluted fractions containing proteins with molecular weight between 13.7 and $67 \mathrm{kDa}$ (Figures 5A,C) confirming the estimate of the molecular weight of the inhibitory factor(s) to be $>10 \mathrm{kDa}$ (Figure 2C). Those active 


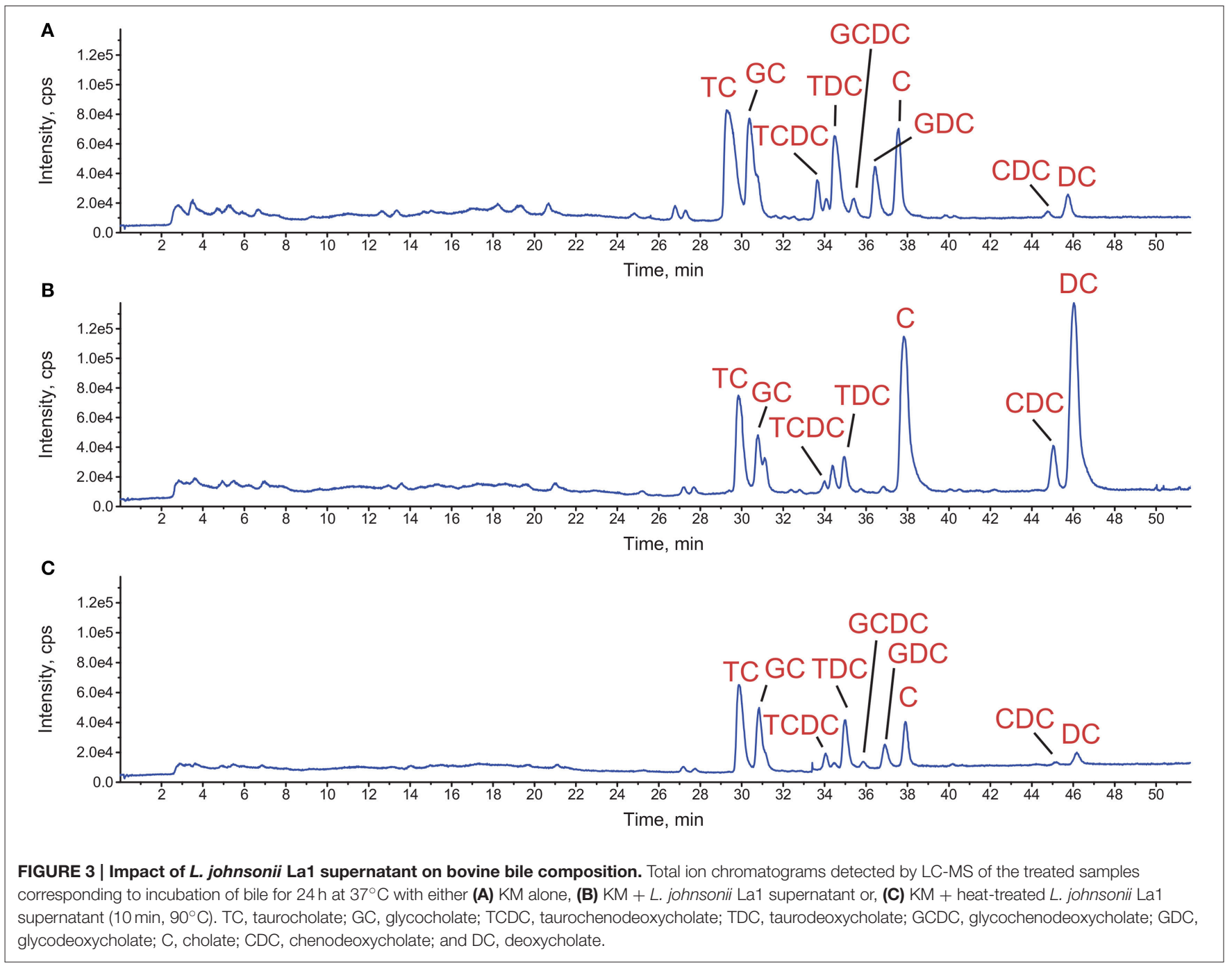

fractions also exhibited a BSH-like activity (Figure 5B), with the most inhibitory fractions showing significantly higher BSHlike activity $(p<0.05)$. Our attempts to further purify elements responsible for the inhibitory activity and/or BSH-like activity by combining steps of ion-exchange, hydrophobic interaction and chromatofocusing chromatographies were unsuccessful, with a rapid loss of activities.

\section{Active Clostridium perfringens BSH in Presence of Tauryl and Glycyl-Bile Salts Mimics the Anti-Giardial Activity of $L$. johnsonii Extracellular Fractions}

To assess the capability of BSH enzymes to promote the bile-mediated anti-Giardia effect, BSH from the bacteria $C$. perfringens was tested for $G$. duodenalis growth inhibition in the presence of bile or pure conjugated bile salts. As notified (Table 3), glycine or taurine conjugated bile salts, TDC, TCDC, GDC, and GCDC have no inhibitory activity on G. duodenalis growth in KM-FCS. In contrast, the addition of $C$. perfringens $\mathrm{BSH}$ to the culture in the presence of the conjugated bile salts led to a remarkable parasite inhibition within the $24 \mathrm{~h}$ of the assay, with inhibition ranges of $95-100 \%$ depending on the conjugated bile salt (Figures 5B, 6). Heat inactivation of $C$. perfringens $\mathrm{BSH}$ $\left(100^{\circ} \mathrm{C}, 5 \mathrm{~min}\right)$ dramatically reduced or even abolished its antiGiardia activity (less than 20\% growth inhibition depending on the conjugated bile salts tested, Figure 6) indicating that parasite growth inhibition depends on $\mathrm{BSH}$ enzymatic activity.

\section{Evidence for the Presence of at least two BSH-Like Enzymes in the L. johnsonii La1 Supernatant}

We investigated whether the BSH-like enzymes annotated from L. johnsonii Lal genome and predicted to be secreted are indeed released into the bacterium culture medium. Highresolution mass spectrometry-based proteomic analysis of $L$. johnsonii Lal extracellular proteins was performed. Three micro gram of proteins from two independent culture supernatants allowed high-confidence identification of 1787 and 1746 peptides (FDR $<1 \%$ at peptide level and only rank 1 peptide identification) providing a total of 164 and 166 protein groups with a minimum of 2 high-confidence peptides per protein, 
A

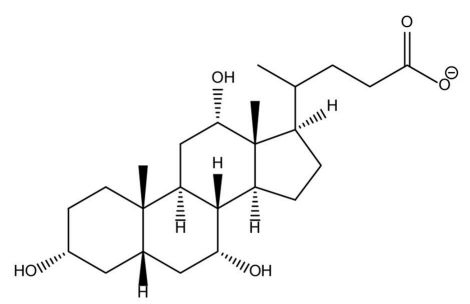

B

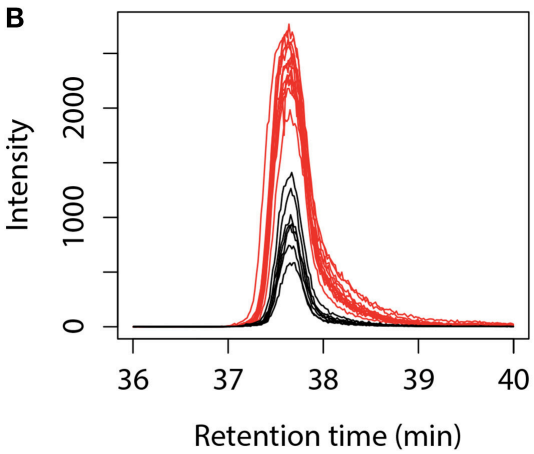

C

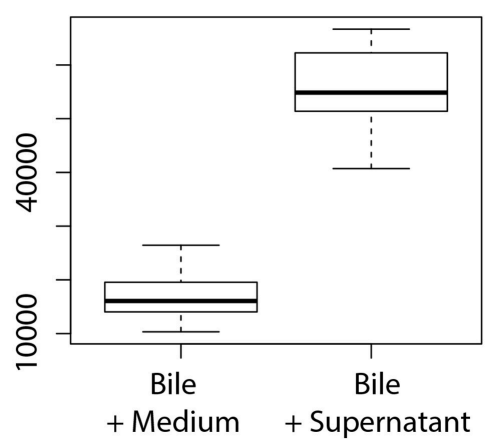

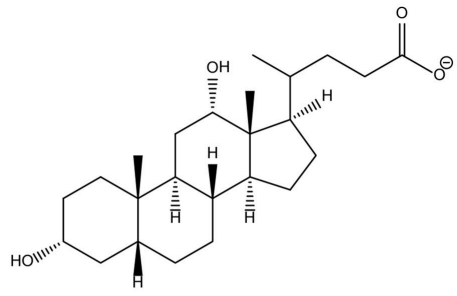
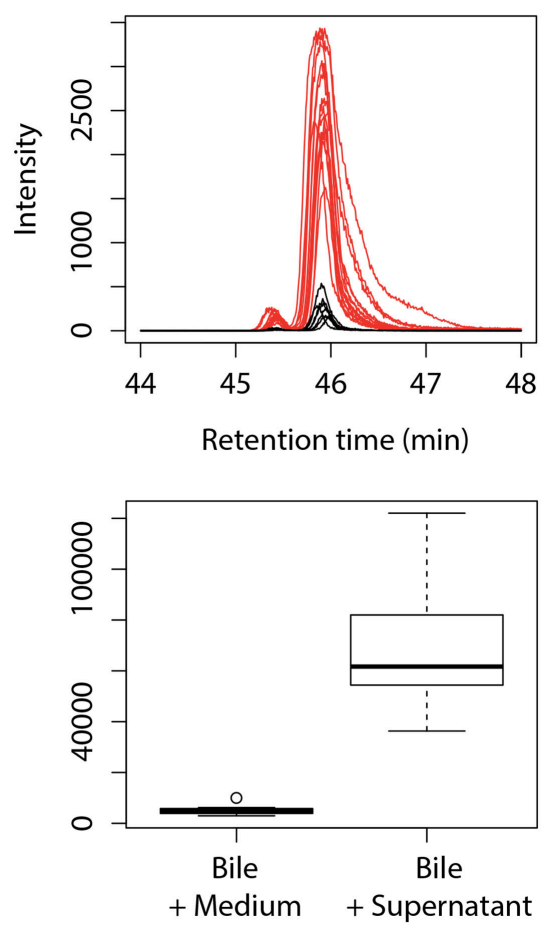

FIGURE 4 | Increase of the non-conjugated salts cholate and deoxycholate content after treatment of bovine bile with $L$. johnsonii La1 supernatant. (A) Structures of cholate ([M-H]- at $\mathrm{m} / \mathrm{z} 407$, left side) and deoxycholate ([M-H]- at $\mathrm{m} / \mathrm{z} 391$, right side). (B,C) Corresponding extracted ion chromatograms (B) and peak area boxplot (C) obtained from bile samples incubated with KM (in black) or L. johnsonii La1 supernatant (in red).

respectively (Table S1) and 147 common proteins between the two replicates (Figure S2). Amongst them, two of the three predicted BSHs, LJ1412 (gi|41583570), and LJ1147 (gi|41583360), were clearly identified by an untargeted/undirected approach in both replicates (Table 4).

\section{DISCUSSION}

Probiotics constitute promising protective alternatives against pathogens, at least as prophylactic agents in various diseases or when used in combination with other therapeutic agents, through a variety of mechanisms relying on immunomodulation properties, competition for nutrients, and habitats, and secretion of active molecules (e.g., antimicrobial peptides, bacteriocins, antibiotics, free fatty acids, hydrogen peroxide; For reviews, Britton and Versalovic, 2008; Travers et al., 2011). In the context of Giardia infections, a number of studies pointed out the importance of the microbiota and the potential role of probiotics in preventing infection and development of disease (Singer and Nash, 2000; Humen et al., 2005; Shukla et al., 2008, 2012; Shukla and Sidhu, 2011; Goyal et al., 2013; Goyal and Shukla, 2013). L. johnsonii La1 (NCC533), Lactobacillus casei MTCC1423, and E. faecium SF68 were consistently identified throughout these studies. In in vivo experiments, those strains were shown to prevent parasite-induced intestinal damage and decrease duration and severity of giardiasis (Humen et al., 2005; Shukla et al., 2008, 2012; Shukla and Sidhu, 2011). Moreover, Pérez et al. (2001) reported inhibitory activity of L. johnsonii La1 on in vitro growth of $G$. duodenalis trophozoites. A partial characterization of the factors involved in the anti-giardial action indicated heat-inactivable low-molecular weight $(<1 \mathrm{kDa})$ components.

Based on this evidence and the potential of probiotics to regulate Giardia proliferation, we aimed to decipher the molecular mechanisms involved in the in vitro anti-giardial 


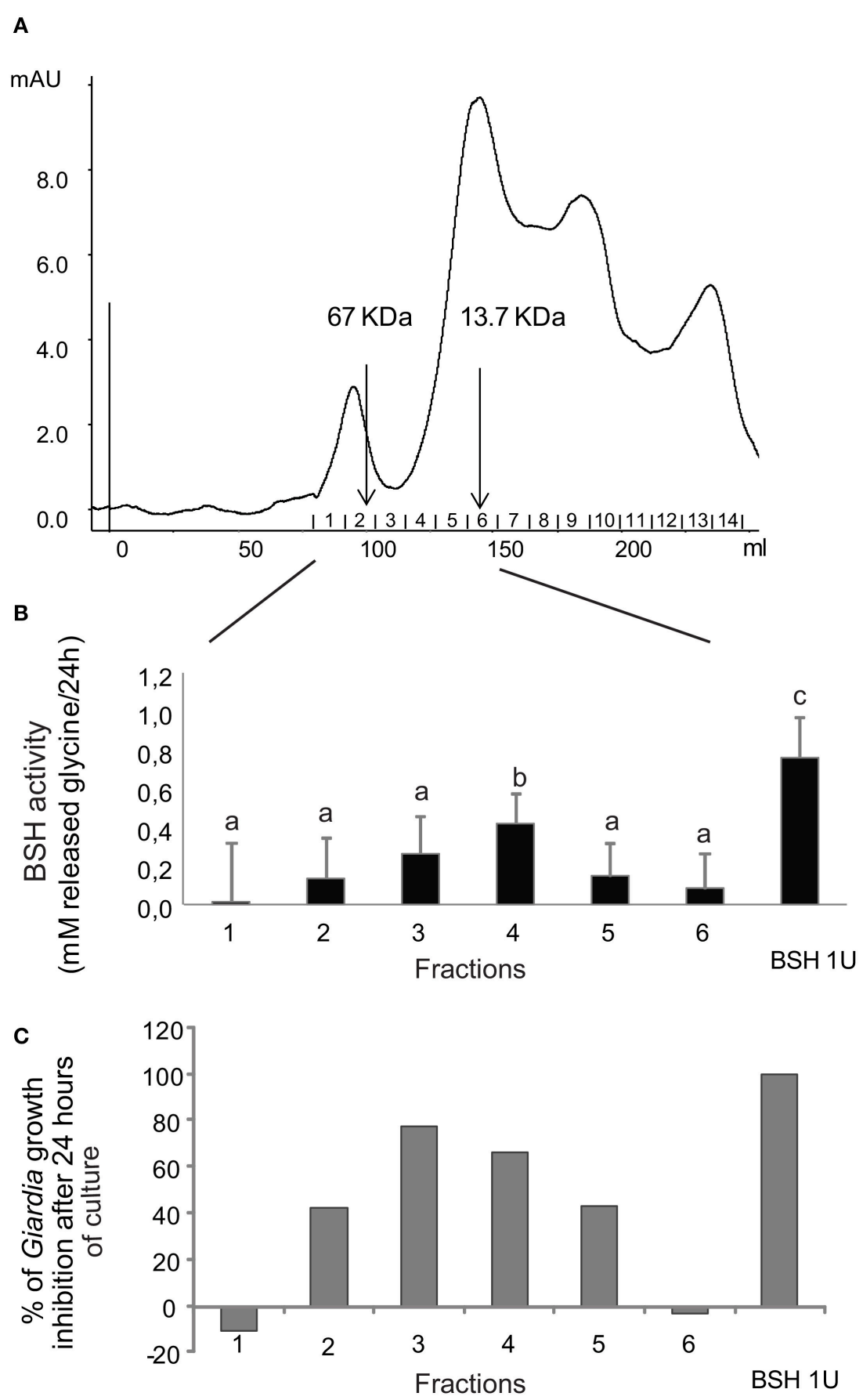

FIGURE 5 | Inhibitory activity against G. duodenalis growth and BSH-like activity co-elute in the same fractions after separation of $L$. johnsonii La1 supernatant by gel filtration chromatography. (A) Chromatography profile. (B) BSH-like activity measured after 24 h of incubation of GDC with the 6 first eluated gel filtration fractions. (C) G. duodenalis growth inhibitory activity after $24 \mathrm{~h}$ of incubation with the same gel filtration chromatography fractions as in B. Letters indicate significant differences between treatments (Kruskal-Wallis, $p<0.05$ ).

activity of L. johnsonii La1. In an experimental assay similar to that published by Pérez et al. (2001), we were able to confirm that $L$. johnsonii La1 supernatants significantly inhibit the proliferation of different strains of $G$. duodenalis in vitro. Pérez et al. (2001) showed that the inhibitory effect of $L$. johnsonii La1 supernatant upon G. duodenalis growth was $\mathrm{pH}$ dependent with a more pronounced inhibitory effect at $\mathrm{pH} 6$ than $\mathrm{pH}$ 7. Inhibitory assays were thus performed at $\mathrm{pH} 6$ instead of $\mathrm{pH} 6.8-7$, the $\mathrm{pH}$ normally used for Giardia culture. The $\mathrm{pH}$ change had a slight effect on parasite proliferation and adhesion properties (Pérez et al., 2001). We also observed no effect on the parasite morphology, motility and viability 
TABLE 3 | Conjugated bile salts in association with $L$. johnsonii La1 supernatant as well as deconjugated bile salts prevent the growth of $G$. duodenalis.

\begin{tabular}{|c|c|c|c|}
\hline & & $\begin{array}{c}\mathrm{KM}+\mathrm{FCS} \mathrm{IC} \mathrm{C}_{50} \\
(\mu \mathrm{M})\end{array}$ & $\begin{array}{l}\mathrm{KM}+\mathrm{FCS}+\text { L. johnsonii } \\
\text { La1 supernatant } \mathrm{IC}_{50}(\mu \mathrm{M})\end{array}$ \\
\hline C & Cholate & $>400$ & $>400$ \\
\hline GC & Glycocholate & $>400$ & $>400$ \\
\hline $\mathrm{TC}$ & Taurocholate & $>400$ & $>400$ \\
\hline $\mathrm{DC}$ & Deoxycholate & $132 \pm 13$ & $117 \pm 13$ \\
\hline GDC & Glycodeoxycholate & $>400$ & $104 \pm 13$ \\
\hline TDC & Taurodeoxycholate & $>400$ & $79 \pm 17$ \\
\hline CDC & Chenodeoxycholate & $147 \pm 15$ & $118 \pm 22$ \\
\hline GCDC & Glycochenodeoxycholate & $>400$ & $110 \pm 11$ \\
\hline TCDC & Taurochenodeoxycholate & $>400$ & $115 \pm 8$ \\
\hline FA & Fusidic acid & $26 \pm 4$ & nd. \\
\hline
\end{tabular}

$I_{50}$ estimations. Various concentrations of taurine-and glycine-conjugated and unconjugated $C, D C$ and $C D C$ bile salts were tested for $G$. duodenalis trophozoite growth inhibition in KM-FCS in the presence or absence of $L$. johnsonii La1 supernatant. $I C_{50}$ values were determined from drug-response curves and expressed as mean $\pm S D$ of at least three independent experiments. nd.: not determined.

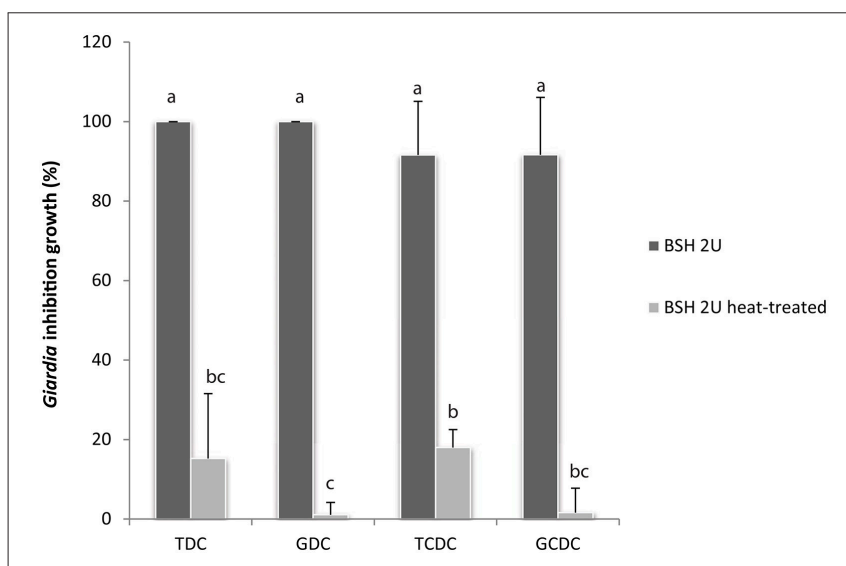

FIGURE 6 | G. duodenalis growth is inhibited by enzymatically active $\mathbf{C}$. perfringens BSH in the presence of conjugated bile salts $(0.2 \mathrm{~g} / \mathrm{L})$. Commercial $\mathrm{C}$. perfringens $\mathrm{BSH}$, enzymatically active or heat-inactivated $\left(100^{\circ} \mathrm{C}, 5 \mathrm{~min}\right.$ ) was added to $\mathrm{G}$. duodenalis culture medium in the presence of either GDC, glycodeoxycholate; GCDC, glycochenodeoxycholate; TDC, taurodeoxycholate or TCDC; taurochenodeoxycholate. Values are the mean \pm $\mathrm{SD}$ of at least three independent experiments. Letters indicate significant differences between treatments (Kruskall-Wallis, $p<0.05$ ).

(Video S1, Figure S1). Furthermore, to avoid an inhibitory activity due to the acidification with lactic acid produced by L. johnsonii La1, bacteria supernatants were neutralized before the assays and appropriate controls were prepared by adding equivalent of lactic acid to fresh medium before $\mathrm{pH}$ adjustment.

Using biochemical fractionations and assays, we were able to document the properties of an active component present in the L. johnsonii Lal supernatant that is inhibitory toward Giardia trophozoites. We first showed that this active(s) component(s) is lactic acid-independent, does not rely on free fatty acids, and is distinct from $\mathrm{H}_{2} \mathrm{O}_{2}$. By using dialysis, ultrafiltrations and combinations of enzymes, we showed that this active(s) component(s) is of peptidic nature, thermosensitive and has a molecular weight $>10 \mathrm{kDa}$. We also demonstrated correlations between this active(s) component(s) inhibiting Giardia and (1) production of deconjugated bile salts toxic to Giardia and (2) BSH activity(ies) of bacterial origin. However, since lactobacilli are known to secrete a large variety of molecules (Lebeer et al., 2008; Cicenia et al., 2014) that could act in concert to bring this antimicrobial effect, and since the active component evidenced by Perez was $<1 \mathrm{kDa}$, we cannot exclude the existence of other factors, secreted or released by L. johnsonii La1, that might also contribute to Giardia inhibition.

We bring here a body of evidence strongly suggesting BSHlike activities in the anti-giardial effect: (1) we observed a conversion of conjugated bile salts into their toxic deconjugated counterparts by $L$. johnsonii La1-supernatant, (2) a similar effect was mimicked by purified Clostridium BSH, (3) we identified two BSHs in L. johnsonii La1 supernatant by mass spectrometry and (4) we measured BSH-like activities in the L. johnsonii La1 fractionated supernatant, with the most enzymatically active fractions mediating the highest toxicity to Giardia.

BSHs (EC 3.5.1.24) are bacterial enzymes known to hydrolyze the amide bond in the C24 position of a wide variety of conjugated bile salts, releasing a deconjugated salt and a taurine or glycine residue (For review, Begley et al., 2006). BSHs are mainly expressed by Gram+ bacteria such as Lactobacillus, Bifodobacterium and Enterococcus species, although some species do not express them. BSH activity allows for the detoxification of conjugated bile salts, which are amphipathic molecules with surfactant properties that can be toxic toward bacterial cells (De Boever and Verstraete, 1999). It is believed that bacterial BSH production is involved in the maintenance of their environment, notably in the small intestine (for the probiotic strains) where bile concentration is at its highest (Begley et al., 2006). This is the first proposal for bacterial $\mathrm{BSH}$ activity regulating parasite survival. Formal identification of the specific BSH(s) implicated in this phenomenon is required. This may be accomplished by recombinant protein production and/or invalidation of the $L$. johnsonii La1 BSH genes (Pridmore et al., 2004). As previous enzymatic studies on BSHs revealed that they may have distinct affinities and specificities for glyco- or tauro- conjugates (For review, Ridlon et al., 2006), it is important to define whether antiGiardia activity is mediated by a single type of BSH with a defined specificity, or by several BSH enzymes with mixed specificities. BSHs from human intestinal lactobacilli usually have the highest affinity for glycine conjugates (Ridlon et al., 2006).

We demonstrate here a two-step mechanism in which a probiotic transforms bile components that could be toxic for itself into components that are toxic to Giardia trophozoites. Giardia tolerance to conjugated bile salts in vitro for concentrations that are toxic to mammalian cell cultures relies on transportmediated processes (Halliday et al., 1995). At least two distinct uptake mechanisms have been described, one $\mathrm{Na}^{+}$-dependent and thiol blocker sensitive for cholyltaurine and the other one $\mathrm{Na}^{+}$- and thiol blocker insensitive for cholylglycine. Both uptake mechanisms are counterbalanced by a rapid efflux 


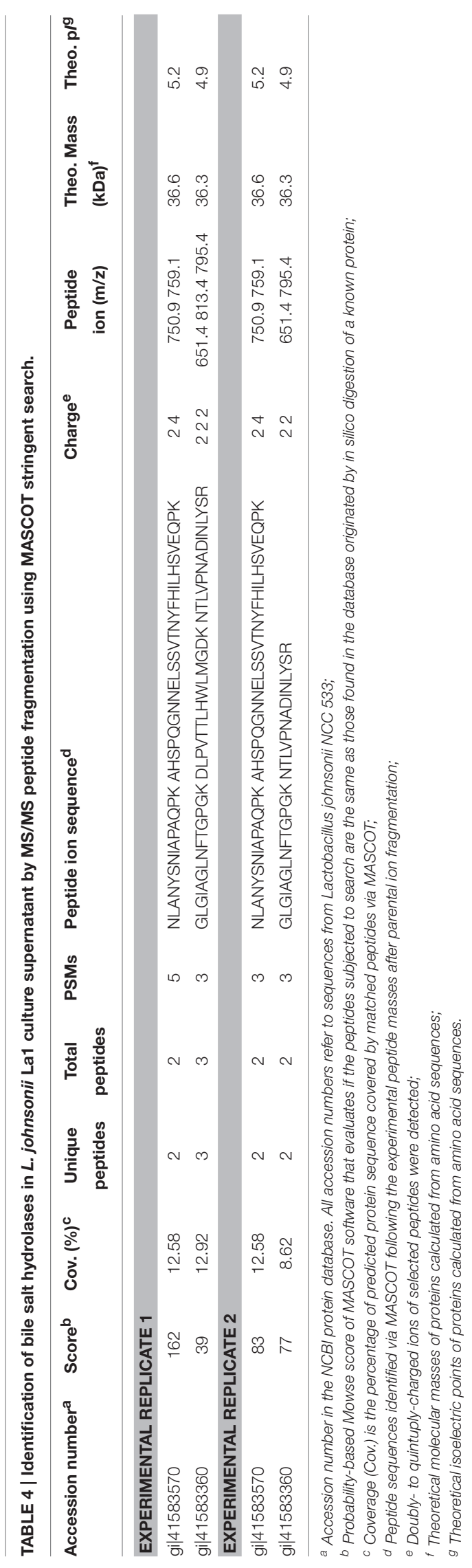

mechanism regulating the intracellular concentration of these detergents to below their toxicity to Giardia (Das et al., 1997). No biotransformation of conjugated bile salts into their deconjugated counterparts by Giardia was observed. The authors suggested that intracellular bile salts may facilitate lipid trafficking and membrane biosynthesis in Giardia. Indeed, Giardia lipid-binding proteins have been shown to have higher affinity for bile salts than fatty acids, suggesting that lipids are incorporated by these binding proteins more readily inside the micelles formed by bile salts than by free fatty acids (de la Guardia et al., 2011). Studies also suggest that conjugated bile salts have a role in promoting Giardia encystation (Gillin, 1987; Gillin et al., 1989), a vital step in the parasite life cycle that enables parasite survival outside of its host. It could be of interest to find out whether such mechanisms can also act on the parasite development cycle (encystation and cyst properties). In this study, experiments were performed using bovine bile instead of using human bile as both species have similar proportions of cholic, desoxycholic, and chenodesoxycholic acids. Besides, human, and bovine bile exhibit an equivalent ratio of glycoconjugated bile salts over their tauroconjugated counterparts (3:1) (Zhong et al., 1998). It can thus be expected a similar inhibitory activity with human bile.

Several studies mentioned briefly the toxic effect of deconjugated bile salts on Giardia (Farthing et al., 1983, 1985, 1987; Gillin, 1987). To our knowledge, our study is the first to demonstrate their dose-dependent anti-giardial activity. Their IC $_{50}$ values are lower than their critical micellar concentrations, indicating that parasite killing is not related to their surfactant properties (e.g., $\mathrm{IC}_{50}$ value for chenodeoxycholate $=0.15 \mathrm{mM}$ and a critical micellar concentration $>7 \mathrm{mM}$ according to the manufacturer). Their mechanism of action remains to be deciphered. Interestingly, their inhibitory activity seems dependent of their hydrophobic properties. No inhibition was observed with the most hydrophilic salt, cholate $\left(\mathrm{IC}_{50}\right.$ value $>400 \mu \mathrm{M}$ ), unlike the more hydrophobic salts, deoxycholate and chenodeoxycholate ( $\mathrm{IC}_{50}$ values of 132 and $147 \mu \mathrm{M}$, respectively). Our results are in line with the previous observation that the bile salt-like antibiotic, fusidic acid, is toxic to Giardia (Farthing and Inge, 1986), which we confirmed $\left(\mathrm{IC}_{50}=26 \mu \mathrm{M}\right)$. However, its taurine and glycine conjugates stimulate parasite growth at low concentrations, while modest growth inhibition occurred at higher concentrations.

In conclusion, we demonstrate that the inhibitory effect exerted in vitro by L. johnsonii La1 supernatant on Giardia growth (1) is dependent on the presence of bile in the growth medium, more precisely bile salts, (2) relies on molecules released by $L$. johnsonii La1 that are of peptidic nature with a molecular weight $>10 \mathrm{kDa}$, (3) is due to the conversion of conjugated bile salts into deconjugated bile salts that are toxic to Giardia, (4) correlates with the presence and activity of BSHlike enzymes from bacterial origin and (5) can be reproduced by addition of $C$. perfringens $\mathrm{BSH}$ to the culture medium. These experimental results point out that BSHs released by $L$. johnsonii La1 would be main facilitators of this anti-parasitic effect. These discoveries open a novel field to fight Giardia based on the 
use of deconjugated bile salts, chemically related drugs or by administration of $\mathrm{BSH}$-releasing probiotic strains.

\section{AUTHOR CONTRIBUTION}

Conceived and designed the experiments: MT, CS, IF, and PG. Performed the experiments: MT, CS, SZ, CD, SoC, RQ, SeC, IF, and PG. Analyzed the data: MT, CS, SZ, CD, RQ, SeC, IF, and PG. Wrote the paper: MT, CS, TA, IF, and PG.

\section{FUNDING}

This work was supported by Région Ile de France, Programme Interdisciplinaire CNRS, "Maladies Infectieuses Emergentes," DIM Maladies Infectieuses, Parasitaires et Nosocomiales Émergentes (progamms $\mathrm{n}^{\circ} 90212$ and DIM 120092), Interdisciplinary Programs of the MNHN (ATMMicroorganismes), the CAPES-COFECUB program [723/11] and $\mathrm{CNPq}$ (Conselho Nacional de Desenvolvimento Cientiífico e Tecnológico).

\section{ACKNOWLEDGMENTS}

We kindly thank Jan Tackezy (Charles University of Prague, Czech Republic) for providing the G. duodenalis HP1 strain, James P. Coleman (Brody School of Medicine, East Caroline University, USA) for providing the recombinant Clostridium perfringens BSH which initiated the study and Pascal Quené (INRA, Jouy en Josas, France) for the L. johnsonii La1 strain. We gratefully acknowledge Lisy Raveendran for her technical

\section{REFERENCES}

Ali, S. A., and Hill, D. R. (2003). Giardia intestinalis. Curr. Opin. Infect. Dis. 16, 453-460. doi: 10.1097/01.qco.0000092817.64370.ab

Ankarklev, J., Jerlström-Hultqvist, J., Ringqvist, E., Troell, K., and Svard, S. G. (2010). Behind the smile: cell biology and disease mechanisms of Giardia species. Nat. Rev. Microbiol. 8, 413-422. doi: 10.1038/nrmicro2317

Ansell, B. R., McConville, M. J., Ma’ayeh, S. Y., Dagley, M. J., Gasser, R. B., Svard, S. G., et al. (2015). Drug resistance in Giardia duodenalis. Biotechnol. Adv. 33(6 Pt 1), 888-901. doi: 10.1016/j.biotechadv.2015.04.009

Baldursson, S., and Karanis, P. (2011). Waterborne transmission of protozoan parasites: review of worldwide outbreaks - an update 2004-2010. Water Res. 45, 6603-6614. doi: 10.1016/j.watres.2011.10.013

Barbosa, J., Costa-de-Oliveira, S., Rodrigues, A. G., and Pina-Vaz, C. (2008). Optimization of a flow cytometry protocol for detection and viability assessment of Giardia lamblia. Travel Med. Infect. Dis. 6, 234-239. doi: 10.1016/j.tmaid.2008.01.004

Bartelt, L. A., and Sartor, R. B. (2015). Advances in understanding Giardia: determinants and mechanisms of chronic sequelae. F1000Prime Rep. 7:62. doi: 10.12703/P7-62

Begley, M., Hill, C., and Gahan, C. G. (2006). Bile salt hydrolase activity in probiotics. Appl. Environ. Microbiol. 72, 1729-1738. doi: 10.1128/AEM.72.3. 1729-1738.2006

Benyacoub, J., Pérez, P. F., Rochat, F., Saudan, K. Y., Reuteler, G., Antille, N., et al. (2005). Enterococcus faecium SF68 enhances the immune response to Giardia intestinalis in mice. J. Nutr. 135, 1171-1176.

Biagini, G. A., Knodler, L. A., Saliba, K. J., Kirk, K., and Edwards, M. R. (2001). $\mathrm{Na}(+)$-dependent $\mathrm{pH}$ regulation by the amitochondriate support and contribution to this study, Amandine Labat for L. johnsonii Lal genotyping, and Peter Roepstorff (Department of Biochemistry and Molecular Biology, University of Southern Denmark, Odense, Denmark) for access to the mass spectrometry facility. The LC/ESI-MS bile acid profiling was performed at the Plateau technique de spectrométrie de masse bio-organique, UMR 7245 Molécules de Communication et Adaptation des Micro-organismes, Muséum National d'Histoire Naturelle, Paris, France.

\section{SUPPLEMENTARY MATERIAL}

The Supplementary Material for this article can be found online at: http://journal.frontiersin.org/article/10.3389/fmicb. 2016.01453

Video S1 | Trophozoites incubated $24 \mathrm{~h}$ in $\mathrm{KM}, \mathrm{pH} 6,0.6 \mathrm{~g} / \mathrm{L}$ bile salts (AVI).

Video S2 | Trophozoites incubated $24 \mathrm{~h}$ in $\mathrm{KM}, \mathrm{pH} 6.0,0.6 \mathrm{~g} / \mathrm{L}$ bile salts and L. johnsonii La1 supernatant (AVI).

Figure S1 | Analysis by flow cytometry of G. duodenalis trophozoite viability after staining with propidium iodide (Word).

Figure S2 | G. duodenalis (WB and HP1 strains) growth inhibition by $L$. johnsonii La1 supernatant in the presence bovine bile from two commercial origins in KM-FCS medium (Word).

Figure S3 | Venn diagram between the two replicates of the L. johnsonii La1 supernatant (Word).

Table S1 | Proteome of L. johnsonii La1 supernatant (2 biological replicates) by MS/MS peptide fragmentation using MASCOT stringent search (XLSX). protozoan parasite Giardia intestinalis. J. Biol. Chem. 276, 29157-29162. doi: 10.1074/jbc.M102728200

Britton, R. A., and Versalovic, J. (2008). Probiotics and gastrointestinal infections. Interdiscip. Perspect. Infect. Dis. 2008:290769. doi: 10.1155/2008/290769

Buret, A. G., Amat, C. B., Manko, A., Beatty, J. K., Halliez, M. C. M., Bhargava, A., et al. (2015). Giardia duodenalis: new research developments in pathophysiology, pathogenesis, and virulence factors. Curr. Trop. Med. Rep. 2 , 110-118. doi: 10.1007/s40475-015-0049-8

Carnaby, S., Katelaris, P. H., Naeem, A., and Farthing, M. J. (1994). Genotypic heterogeneity within Giardia lamblia isolates demonstrated by M13 DNA fingerprinting. Infect. Immun. 62, 1875-1880.

Charneau, S., Junqueira, M., Costa, C., Pires, D., Fernandes, E., Bussacos, A., et al. (2007). The saliva proteome of the blood-feeding insect Triatoma infestans is rich in platelet-aggregation inhibitors. Int. J. Mass Spectrom. 268, 265-276. doi: 10.1016/j.ijms.2007.05.004

Cicenia, A., Scirocco, A., Carabotti, M., Pallotta, L., Marignani, M., and Severi, C. (2014). Postbiotic activities of lactobacilli-derived factors. J. Clin. Gastroenterol. 48(Suppl. 1), S18-S22. doi: 10.1097/MCG.0000000000 000231

Cotton, J. A., Beatty, J. K., and Buret, A. G. (2011). Host parasite interactions and pathophysiology in Giardia infections. Int. J. Parasitol. 41, 925-933. doi: 10.1016/j.ijpara.2011.05.002

Das, S., Schteingart, C. D., Hofmann, A. F., Reiner, D. S., Aley, S. B., and Gillin, F. D. (1997). Giardia lamblia: evidence for carrier-mediated uptake and release of conjugated bile acids. Exp. Parasitol. 87, 133-141.

De Boever, P., and Verstraete, W. (1999). Bile salt deconjugation by Lactobacillus plantarum 80 and its implication for bacterial toxicity. J. Appl. Microbiol. 87, 345-352. 
de la Guardia, R. D., Lopez, M. B., Burgos, M., and Osuna, A. (2011). Purification and characterization of a protein capable of binding to fatty acids and bile salts in Giardia lamblia. J. Parasitol. 97, 642-647. doi: 10.1645/GE-2469.1

Denou, E., Pridmore, R. D., Ventura, M., Pittet, A. C., Zwahlen, M. C., Berger, B., et al. (2008). The role of prophage for genome diversification within a clonal lineage of Lactobacillus johnsonii: characterization of the defective prophage LJ771. J. Bacteriol. 190, 5806-5813. doi: 10.1128/JB.01802-07

Duncombe, W. G. (1964). The colorimetric micro-determination of non-esterified fatty acids in plasma. Clin. Chim. Acta 9, 122-125.

Farthing, M. J., and Inge, P. M. (1986). Antigiardial activity of the bile salt-like antibiotic sodium fusidate. J. Antimicrob. Chemother. 17, 165-171.

Farthing, M. J., Inge, P. M., and Pearson, R. M. (1987). Effect of D-propranolol on growth and motility of flagellate protozoa. J. Antimicrob. Chemother. 20, 519-522.

Farthing, M. J., Keusch, G. T., and Carey, M. C. (1985). Effects of bile and bile salts on growth and membrane lipid uptake by Giardia lamblia. Possible implications for pathogenesis of intestinal disease. J. Clin. Invest. 76, 1727-1732. doi: $10.1172 /$ JCI112162

Farthing, M. J., Varon, S. R., and Keusch, G. T. (1983). Mammalian bile promotes growth of Giardia lamblia in axenic culture. Trans. R. Soc. Trop. Med. Hyg. 77, 467-469.

Franzén, O., Jerlstrom-Hultqvist, J., Castro, E., Sherwood, E., Ankarklev, J., Reiner, D. S., et al. (2009). Draft genome sequencing of Giardia intestinalis assemblage B isolate GS: is human giardiasis caused by two different species? PLoS Pathog. 5:e1000560. doi: 10.1371/journal.ppat.1000560

Gardner, T. B., and Hill, D. R. (2001). Treatment of Giardiasis. Clin. Microbiol. Rev. 14, 114-128. doi: $10.1128 / \mathrm{cmr} \cdot 14.1 .114-128.2001$

Gillin, F. D. (1987). Giardia lamblia: the role of conjugated and unconjugated bile salts in killing by human milk. Exp. Parasitol. 63, 74-83.

Gillin, F. D., Boucher, S. E., Rossi, S. S., and Reiner, D. S. (1989). Giardia lamblia: the roles of bile, lactic acid, and $\mathrm{pH}$ in the completion of the life cycle in vitro. Exp. Parasitol. 69, 164-174.

Gillin, F. D., Gault, M. J., Hofmann, A. F., Gurantz, D., and Sauch, J. F. (1986). Biliary lipids support serum-free growth of Giardia lamblia. Infect. Immun. 53, 641-645.

Goyal, N., Rishi, P., and Shukla, G. (2013). Lactobacillus rhamnosus GG antagonizes Giardia intestinalis induced oxidative stress and intestinal disaccharidases: an experimental study. World J. Microbiol. Biotechnol. 29, 1049-1057. doi: 10.1007/s11274-013-1268-6

Goyal, N., and Shukla, G. (2013). Probiotic Lactobacillus rhamnosus GG modulates the mucosal immune response in Giardia intestinalis-infected BALB/c mice. Dig. Dis. Sci. 58, 1218-1225. doi: 10.1007/s10620-012-2503-y

Griffiths, W. J., and Sjovall, J. (2010). Bile acids: analysis in biological fluids and tissues. J. Lipid Res. 51, 23-41. doi: 10.1194/jlr.R001941-JLR200

Grill, J., Cayuela, C., Antoine, J., and Schneider, F. (2000). Isolation and characterization of a Lactobacillus amylovorus mutant depleted in conjugated bile salt hydrolase activity: relation between activity and bile salt resistance. J. Appl. Microbiol. 89, 553-563. doi: 10.1046/j.1365-2672.2000. 01147.x

Halliday, C. E., Inge, P. M., and Farthing, M. J. (1995). Characterization of bile salt uptake by Giardia lamblia. Int. J. Parasitol. 25, 1089-1097.

Halliez, M. C., and Buret, A. G. (2013). Extra-intestinal and long term consequences of Giardia duodenalis infections. World J. Gastroenterol. 19, 8974-8985. doi: 10.3748/wjg.v19.i47.8974

Humen, M. A., De Antoni, G. L., Benyacoub, J., Costas, M. E., Cardozo, M. I., Kozubsky, L., et al. (2005). Lactobacillus johnsonii La1 antagonizes Giardia intestinalis in vivo. Infect. Immun. 73, 1265-1269. doi: 10.1128/IAI.73.2.12651269.2005

Itaya, K., and Ui, M. (1965). Colorimetric determination of free fatty acids in biological fluids. J. Lipid Res. 6, 16-20.

Kamada, N., Chen, G. Y., Inohara, N., and Núñez, G. (2013). Control of pathogens and pathobionts by the gut microbiota. Nat. Immunol. 14, 685-690. doi: 10.1038/ni.2608

Keister, D. B. (1983). Axenic culture of Giardia lamblia in TYI-S-33 medium supplemented with bile. Trans. R. Soc. Trop. Med. Hyg. 77, 487-488.

Lalle, M. (2010). Giardiasis in the post genomic era: treatment, drug resistance and novel therapeutic perspectives. Infect. Disord. Drug Targets 10, 283-294. doi: $10.2174 / 187152610791591610$
Lane, S., and Lloyd, D. (2002). Current trends in research into the waterborne parasite Giardia. Crit. Rev. Microbiol. 28, 123-147. doi: 10.1080/1040840291046713

Lebeer, S., Vanderleyden, J., and De Keersmaecker, S. C. (2008). Genes and molecules of lactobacilli supporting probiotic action. Microbiol. Mol. Biol. Rev. 72, 728-764, doi: 10.1128/MMBR.00017-08

Leitsch, D., Burgess, A. G., Dunn, L. A., Krauer, K. G., Tan, K., Duchêne, M., et al. (2011). Pyruvate:ferredoxin oxidoreductase and thioredoxin reductase are involved in 5-nitroimidazole activation while flavin metabolism is linked to 5-nitroimidazole resistance in Giardia lamblia. J. Antimicrob. Chemother. 66, 1756-1765. doi: $10.1093 / \mathrm{jac} / \mathrm{dkr} 192$

Mitamura, K., Hori, N., Iida, T., Hofmann, A. F., and Ikegawa, S. (2011). Identification of bile acid S-acyl glutathione conjugates in rat bile by liquid chromatography/electrospray ionization-linear ion trap mass spectrometry. Steroids 76, 68-77. doi: 10.1016/j.steroids.2010.09.002

Mons, C., Dumetre, A., Gosselin, S., Galliot, C., and Moulin, L. (2009). Monitoring of Cryptosporidium and Giardia river contamination in Paris area. Water Res. 43, 211-217. doi: 10.1016/j.watres.2008.10.024

Morrison, H. G., McArthur, A. G., Gillin, F. D., Aley, S. B., Adam, R. D., Olsen, G. J., et al. (2007). Genomic minimalism in the early diverging intestinal parasite Giardia lamblia. Science 317, 1921-1926. doi: 10.1126/science. 114383

Nair, P. P., Gordon, M., and Reback, J. (1967). The enzymatic cleavage of the carbon-nitrogen bond in 3-alpha, 7-alpha, 12-alpha-trihydroxy-5-beta-cholan24-oylglycine. J. Biol. Chem. 242, 7-11.

Paget, T., Maroulis, S., Mitchell, A., Edwards, M. R., Jarroll, E. L., and Lloyd, D. (2004). Menadione kills trophozoites and cysts of Giardia intestinalis. Microbiology 150(Pt 5), 1231-1236. doi: 10.1099/mic.0.26836-0

Pérez, P. F., Minnaard, J., Rouvet, M., Knabenhans, C., Brassart, D., De Antoni, G. L., et al. (2001). Inhibition of Giardia intestinalis by extracellular factors from Lactobacilli: an in vitro study. Appl. Environ. Microbiol. 67, 5037-5042. doi: 10.1128/AEM.67.11.5037-5042.2001

Perwaiz, S., Tuchweber, B., Mignault, D., Gilat, T., and Yousef, I. M. (2001). Determination of bile acids in biological fluids by liquid chromatographyelectrospray tandem mass spectrometry. J. Lipid Res. 42, 114-119.

Pridmore, R. D., Berger, B., Desiere, F., Vilanova, D., Barretto, C., Pittet, A. C., et al. (2004). The genome sequence of the probiotic intestinal bacterium Lactobacillus johnsonii NCC 533. Proc. Natl. Acad. Sci. U.S.A. 101, 2512-2517. doi: 10.1073/pnas.0307327101

Pridmore, R. D., Pittet, A. C., Praplan, F., and Cavadini, C. (2008). Hydrogen peroxide production by Lactobacillus johnsonii NCC 533 and its role in antiSalmonella activity. FEMS Microbiol. Lett. 283, 210-215. doi: 10.1111/j.15746968.2008.01176.x

Queiroz, R. M., Charneau, S., Motta, F. N., Santana, J. M., Roepstorff, P., and Ricart, C. A. (2013). Comprehensive proteomic analysis of Trypanosoma cruzi epimastigote cell surface proteins by two complementary methods. J. Proteome Res. 12, 3255-3263. doi: 10.1021/pr400110h

Ridlon, J. M., Kang, D. J., and Hylemon, P. B. (2006). Bile salt biotransformations by human intestinal bacteria. J. Lipid Res. 47, 241-259. doi: 10.1194/jlr.R500013-JLR200

Rohrer, L., Winterhalter, K. H., Eckert, J., and Kohler, P. (1986). Killing of Giardia lamblia by human milk is mediated by unsaturated fatty acids. Antimicrob. Agents Chemother. 30, 254-257.

Shukla, G., Devi, P., and Sehgal, R. (2008). Effect of Lactobacillus casei as a probiotic on modulation of giardiasis. Dig. Dis. Sci. 53, 2671-2679. doi: 10.1007/s10620007-0197-3

Shukla, G., and Sidhu, R. K. (2011). Lactobacillus casei as a probiotic in malnourished Giardia lamblia-infected mice: a biochemical and histopathological study. Can. J. Microbiol. 57, 127-135. doi: 10.1139/w 10-110

Shukla, G., Sidhu, R. K., and Verma, A. (2012). Restoration of anthropometric, biochemical and histopathological alterations by Lactobacillus casei supplementation in Giardia intestinalis infected renourished BALB/c mice. Antonie Van Leeuwenhoek 102, 61-72. doi: 10.1007/s10482-012-9713-3

Singer, S. M., and Nash, T. E. (2000). The role of normal flora in Giardia lamblia infections in mice. J. Infect. Dis. 181, 1510-1512. doi: 10.1086/315409

Smith, C. A., Want, E. J., O’Maille, G., Abagyan, R., and Siuzdak, G. (2006). XCMS: processing mass spectrometry data for metabolite profiling using nonlinear 
peak alignment, matching, and identification. Anal. Chem. 78, 779-787. doi: $10.1021 /$ ac051437y

Tancrède, C. (1992). Role of human microflora in health and disease. Eur. J. Clin. Microbiol. Infect. Dis. 11, 1012-1015.

Tian, X. F., Shen, H. E., Li, J., Chen, Y., Yang, Z. H., and Lu, S. Q. (2010). The effects of dihydroartemisinin on Giardia lamblia morphology and cell cycle in vitro. Parasitol. Res. 107, 369-375. doi: 10.1007/s00436-010-1872-4

Travers, M. A., Florent, I., Kohl, L., and Grellier, P. (2011). Probiotics for the control of parasites: an overview. J. Parasitol. Res. 2011:610769. doi: $10.1155 / 2011 / 610769$

Upcroft, P., and Upcroft, J. A. (2001). Drug targets and mechanisms of resistance in the anaerobic protozoa. Clin. Microbiol. Rev. 14, 150-164. doi: 10.1128/CMR.14.1.150-164.2001

Veenemans, J., Mank, T., Ottenhof, M., Baidjoe, A., Mbugi, E. V., Demir, A. Y., et al. (2011). Protection against diarrhea associated with Giardia intestinalis is lost with multi-nutrient supplementation: a study in Tanzanian children. PLoS Negl. Trop. Dis. 5:e1158. doi: 10.1371/journal.pntd. 0001158
Ventura, M., Canchaya, C., Pridmore, R. D., and Brussow, H. (2004). The prophages of Lactobacillus johnsonii NCC 533: comparative genomics and transcription analysis. Virology 320, 229-242. doi: 10.1016/j.virol.2003.11.034

Zhong, W., Millsap, K., Bialkowska-Hobrzanska, H., and Reid, G. (1998). Differentiation of Lactobacillus Species by Molecular Typing. Appl. Environ. Microbiol. 64, 2418-2423.

Conflict of Interest Statement: The authors declare that the research was conducted in the absence of any commercial or financial relationships that could be construed as a potential conflict of interest.

Copyright (c) 2016 Travers, Sow, Zirah, Deregnaucourt, Chaouch, Queiroz, Charneau, Allain, Florent and Grellier. This is an open-access article distributed under the terms of the Creative Commons Attribution License (CC BY). The use, distribution or reproduction in other forums is permitted, provided the original author(s) or licensor are credited and that the original publication in this journal is cited, in accordance with accepted academic practice. No use, distribution or reproduction is permitted which does not comply with these terms. 